\title{
A New Hybrid Model Based on an Intelligent Optimization Algorithm and a Data Denoising Method to Make Wind Speed Predication
}

\author{
Ping Jiang and Qingli Dong \\ School of Statistics, Dongbei University of Finance and Economics, Dalian, Liaoning 116025, China \\ Correspondence should be addressed to Qingli Dong; isaacdon525@gmail.com
}

Received 23 April 2015; Revised 31 May 2015; Accepted 11 June 2015

Academic Editor: Xiaosong $\mathrm{Hu}$

Copyright (c) 2015 P. Jiang and Q. Dong. This is an open access article distributed under the Creative Commons Attribution License, which permits unrestricted use, distribution, and reproduction in any medium, provided the original work is properly cited.

\begin{abstract}
To mitigate the increase of anxiety resulting from the depletion of fossil fuels and destruction of the ecosystem, wind power, as the most common renewable energy, is a flourishing industry. Thus, accurate wind speed forecasting is critical for the efficient function of wind farms. However, affected by complicated influence factors in meteorology and volatile physical property, wind speed forecasting is difficult and challenging. Based on previous research efforts, an intelligent hybrid model was proposed in this paper in an attempt to tackle this difficult task. First, wavelet transform was utilized to extract the main components of the original wind speed data while eliminating noise. To make better use of the back-propagation artificial neural network, the initial parameters of the network are substituted with optimized ones, which are achieved by using the artificial fish swarm algorithm (AFSA), and the final combination model is employed to conduct wind speed forecasting. A series of data are collected from four different observation sites to test the validity of the proposed model. Through comprehensive comparison with the traditional models, the experiment results clearly indicate that the proposed hybrid model outperforms the traditional single models.
\end{abstract}

\section{Introduction}

With fossil fuels gradually drying up and the aftermath of the polluted environment becoming increasingly obvious, the world is sparing no effort to explore and exploit renewable energy for future power generation [1]. However, intermittence is the nature of the most renewable energy sources, which presents a great challenge to maintain and ensure the stability and reliability of power network [2]. Thus, effective energy storage technologies play a paramount role in mitigating the volatility [3]. As key ingredients, electric vehicles (EVs) would be widely regarded as an integral part of such a renewable energy system if they use electricity generated by a renewable energy source [4]. Besides, oil and coal supply uncertainty, growing mobility demand, and increasingly stringent regulations on pollutants and carbon footprints are expediting a paradigm shift towards sustainable power system and transportation $[5,6]$. Until now, even though numerous clean energy technologies, such as plug-in hybrid electric vehicles (PHEVs) [7], battery management system (BMS) [8], and battery electric vehicles (BEVs) [9], are being widely deployed and developed to significantly reduce the fuel consumption and the carbon emission throughout the world, they also increase the demand of power in the meantime. Thus, expanding investment on exploration and exploitation of the supplement of traditional energy is badly needed.

Wind power, the most recognized renewable energy source (RES), definitely possesses the prominent potential to become the resource of electricity, which plays a fundamental and indispensable role in social modernization [10]. Among different types of renewable energy, such as solar, wind, geothermal, and tidal energy, wind power is the fastestdeveloping and the most widely used one and also meets the demand for large scale commercial exploitation, owing to the maturity of the technology [11].

Currently, the technology of wind power has attracted worldwide attention and occupied a considerable share in the proportion of energy construction. Many countries take the development of wind power as an effective action to improve their domestic energy structures, preserve the ecological 
environment, alleviate environmental pollution, and even integrate it into the strategic development planning for the entire power system.

China, with such a great population, will soon replace America as the leading energy consuming country [12]. Depending on a series of positive policies, the wind power industry has stepped into rapid development and the present scale is exciting. The capacity of exploitable wind power is on rise year by year as technology progresses. According to a study started by the National Energy Administration (NEA), in the national wind power long-term plan, it is explicitly proposed that, to 2020, the national wind power installed capacity goal is 20,000,000 kilowatts [13]. In order to achieve such a capacity of power, nearly 708 million tons coal are needed according to the internationally accepted conversion standard, and the accompanying carbon emission even reaches to 480 million tons. Therefore, developing wind power efficiently does not only have economic significance, but also high ecological value.

With such a large capacity of wind power, the integration of wind power becomes an issue. Furthermore, high randomness of wind brings a big problem for a wind farm to provide electric grids to meet its necessary need of power, and this characteristic of wind power is a big challenge to traditional power system which has a relatively mature operation mode. Hence, accurate wind speed forecasting, as a necessary step in wind power integration, is particularly important, and these are the contributions of this paper.

There are three acknowledged methods for forecasting wind speed, including long-term prediction, medium-term prediction, and short-term prediction [14]. Briefly, longterm forecasting is used in the process of site selection for wind farms and in the maintenance and overhaul of the power network. When researching the dynamic economic dispatch scheme in a grid-connected wind power system, the medium-term forecasting of wind that usually spans a few days or weeks comes in handy. As for the latter method, it mostly works to meet the control requirements of wind turbines. Overall, apparently, forecast error will become greater as the forecast horizon increases. Thus, the accuracy of short-term forecasting is particularly important, for it lays the foundation of the renewable energy program, security evaluation, optimal dispatching model, and other critical decisions in power network dispatching.

Until now, many scientists have devoted research efforts to short-term wind speed forecasting, and various achievements have been successfully applied. Numerical simulation, as early results, is usually used to construct mathematical models to simulate the dynamical physical behaviour of wind. These models include many linear, nonlinear, difference, and differential equations related with many conditions of wind, and those numerical solutions are usually achieved by calculation and iterative methods. However, because of the astatic and chaotic characteristics of the factors involved in the numerical simulation, as well as many uncertainties in the forecasting process, the results of these methods usually cannot meet the requirements of precision [15].

To solve these difficulties, many new models have been applied, which can roughly be categorized into physical, such as the Numerical Weather Prediction systems (NWPs), statistical, containing linear methods such as the Auto Regressive model (AR), Moving Average (MA), Auto Regressive Integrated Moving Average (ARIMA) model, and intelligent methods, which come from artificial intelligence and machine learning fields, such as the artificial neural networks (ANN) [16], and even by hybrid approach methods, which are combination of statistical and physical methods that use the analysis of a time series [17].

ARIMA, as a traditional statistical approach, has been proposed by some researchers to forecast wind speed, though it should be improved when dealing with the volatility and chaotic characteristics of wind speed. Ernst et al. supplied a method using past power measurements and meteorological predictions of the data of wind speed and direction interpolated at a wind farm [18]. The Grey prediction model $\operatorname{GM}(1, n)$, as a novel method for wind speed forecasting, as well as a wind power predictor, that reflects a Grey model that has a differential equation with a single variable, was provided by El-Fouly. The structure of the artificial neural network, which was applied for forecasting the mean monthly wind speed in regions of Cyprus, is discussed by Baran, though the data were not sufficient [19]. In addition, a hybrid model that combined the Kalman filter (KF) with the artificial neural network was proposed based on an ARIMA model to further enhance the forecasting accuracy of wind speed. Sideratos and Hatziargyriou [20] employed a hybrid method to forecast wind speed in which three independent algorithms had been adopted. They were the Seasonal Adjustment Algorithm, the Exponential Smoothing Method (ESM), and the Radial Basis Function (RBF) neural network, and the final results indicated that the suggested hybrid method had satisfactory performance.

Back-propagation, as one of the most common learning algorithms among the artificial neural networks, has obvious advantages in nonlinear model fitting and predication because of its unique characteristic in terms of high tolerance with data errors. From the operational principle of the learning algorithm of the BPANN model, it can be observed that the initial weights and thresholds of the back-propagation neural network have a serious impact on the accuracy of the model [21].

In addition to the model, the characteristic of the initial data can also affect the final forecasting of the wind speed. In fact, the original data from the wind farm usually cannot be used in the model directly because there is much chaotic and scrambled information [22]; then, the WT is employed in this work to preprocess the data of ten-minute wind speed to eliminate the high frequency disturbance. For the sake of improving the accuracy of wind speed forecasting further, a new optimization algorithm called the artificial fish swarm algorithm (AFSA) is proposed in this paper. The AFSA adapted here is used to find the proper parameters of the initial back-propagation artificial neural network, whose input is the processed ten-minute wind speed data. The final results of the data showed that the hybrid optimal AFSA and BPANN method can perform better in short-term wind speed forecasting compared with previous models. 
The rest of this paper is organized as follows. The analysed data are described in Section 2. A general description of relative methods is provided in Section 3. In Section 4, the hybrid optimal model is discussed in detail; specifically, the process of selection of the optimal parameters of the propagation and the overall structure of the WAFSA-BPANN model will be presented and introduced explicitly. In Section 5, the simulation of the model and the evaluation of its final performance of wind speed forecasting are discussed in detail. The conclusions of this paper are presented in Section 6 .

\section{The Sources and Characteristics of the Data Set}

To meet the demands of the model proposed in this paper, a variety of data were collected from turbines of wind farms from the Shandong Peninsula of China. Owing to its abundant sources of wind from the sea, which are richly endowed by nature, the Shandong Peninsula plays a more and more important role within the entire large sector of the Chinese wind power structure. However, it is difficult to forecast and evaluate wind speed because there are too many factors that can affect the changing of the wind speed. Our effective and reliable method is to find out the valuable and useful information, which the proposed model can then utilize to display the forecasting through the historical data.

The data used in this paper are collected from three different turbines of a wind farm. To ensure the fairness and consider the potential periodicity of seasons, the involved data series of wind speed cover four seasons in 2011. To be specific, data from four representative months in different seasons are chosen to implement the simulation and forecasting, namely, January, May, July, and September. For instance, ten-minute wind speed data series from January 1 to January 31 in 2011 are collected. Each day, the data series span from 00:00 to 23:50, and the wind speed data are recorded every ten minutes. Figure 1 shows the whole profile of the wind speed data series spanning four seasons in one of the three chosen observation sites.

From Figure 1, the obvious feature of fluctuation and undulation of the original wind speed data series can be observed, and there are no rules that may be utilized to conduct forecasting through simple estimation. Besides, the results of parameter estimation and testing indicate that wind speed data series fit the Weibull distribution. Though many models can tolerate the instability of the original data, the accuracy of the forecasting models can still be impaired by the noise and disturbance suffused in them. Thus, there are active imperious demands to preprocess the initial data series to achieve a relatively smooth time series. Herein, wavelet transform was proposed to dispose the wind speed data series firstly, and preprocessed data were applied to execute the hybrid model, which will be discussed in the next section.

\section{Description of Relative Models}

Several methods are referred to in this paper, such as wavelet transform, back-propagation artificial neural network, and artificial fish swarm algorithm; each of them will be explained briefly below.

3.1. Wavelet Transform (WT). Wavelet transform is a type of denoising method that can decompose signals with different frequencies. The essence of WT is to transform a one-dimensional signal in the time domain into a twodimensional time-frequency signal. WT can carry out the multiresolution analysis, which indicates that WT has a high time resolution and low frequency resolution in the high frequency part; in the low frequency part, WT has a high frequency resolution and low time resolution [23]. The basic idea of WT is to approach the primitive function by using two of the above functions. Assume that the signal $X(t)$ is the square integrable function; then, the WT of the signal is the inner product with $\psi_{(\alpha, \tau)}(t)$ :

$$
\begin{aligned}
\mathrm{WT}_{X}(\alpha, \tau) & =\frac{1}{\sqrt{\alpha}} \int X(t) \psi^{*}\left(\frac{t-\tau}{\alpha}\right) d t \\
& =\left\langle X(t), \psi_{(\alpha, \tau)}(t)\right\rangle,
\end{aligned}
$$

where $\alpha$ and $\tau$ are the scaling and shift factor, respectively, and $\psi^{*}(t)$ is the adjoin function of $\psi(t)$.

The basic steps of WT are as follows.

Step 1 (Decompose the original signal). Choose the wavelet basis and decomposition hierarchy and calculate coefficients of wavelet decomposition in each layer.

Step 2 (Address the threshold value of high frequency coefficients). Choose a threshold value for each decomposition hierarchy and process the high frequency coefficients to eliminate the noise centred on the high frequency part.

Step 3 (Carry out wavelet reconstruction of signal). Carry out the wavelet reconstruction for low frequency coefficients and high frequency coefficients such that the threshold value is processed quantitatively [24].

Through the wavelet transform, the high frequency wind speed data series with significant noise is eliminated, and the residual of the subseries is combined to substitute the original data. From Figure 2, it is obvious that the denoising data series is more smooth and stable than the original data. The simulation and forecasting results upon the preprocessed data employed in this paper also indicate the necessity and superiority of the wavelet transform.

It should be noted here that the entire preprocessed data are only used to train the hybrid model involved hereinafter. To be specific, wind speed data during the whole month are employed to use the wavelet transform, and the final forecasting of the next week is achieved from the original real wind speed data.

3.2. Back-Propagation Artificial Neural Network (BPANN). The schematic of the BP artificial neural network is plotted in Figure 3(a). Applying the classic back-propagation optimal algorithm, the BPANN is widely used in multidomains. This particular BP neural network has three layers: the input layer, 

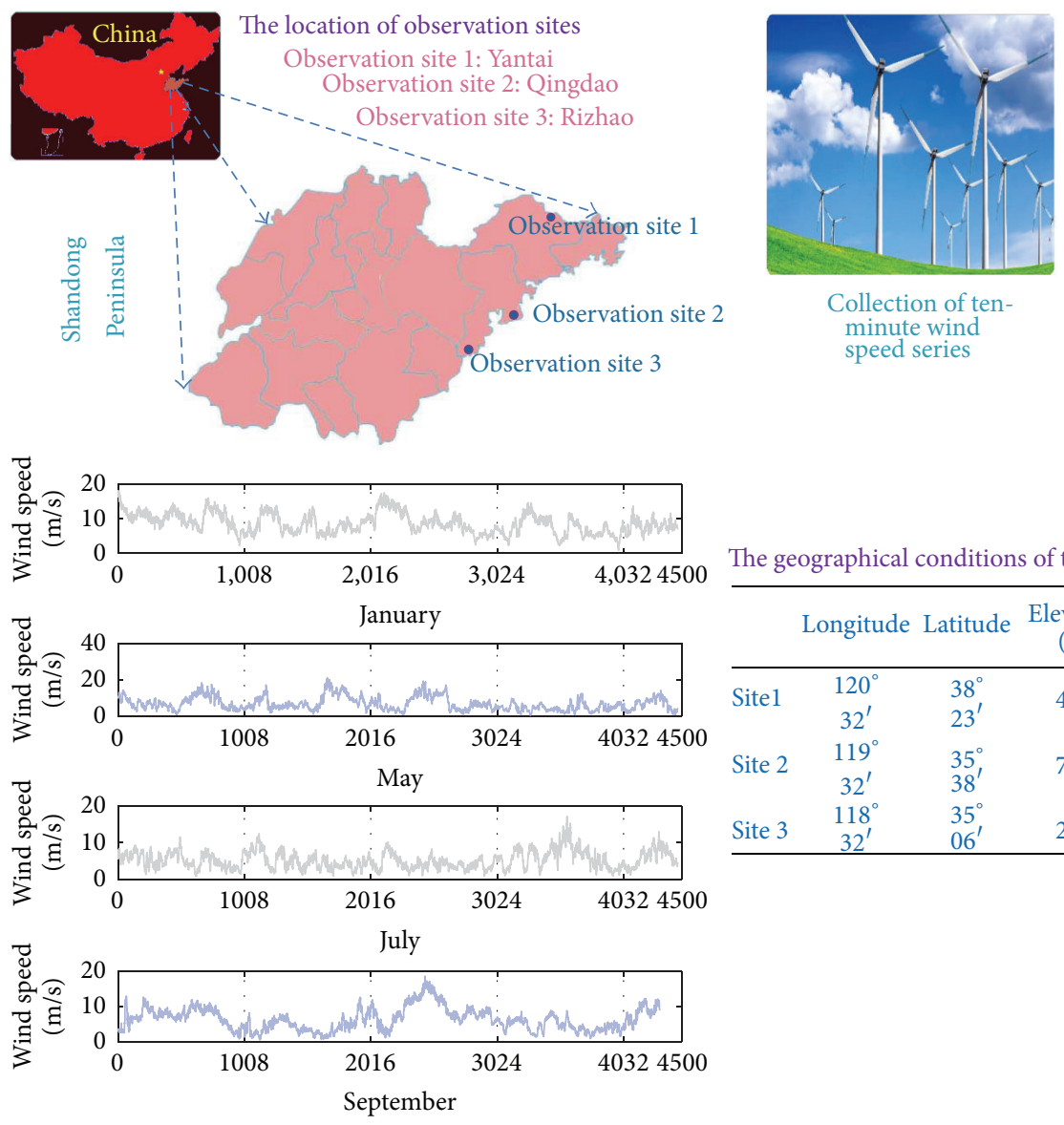

The geographical conditions of three observation sites
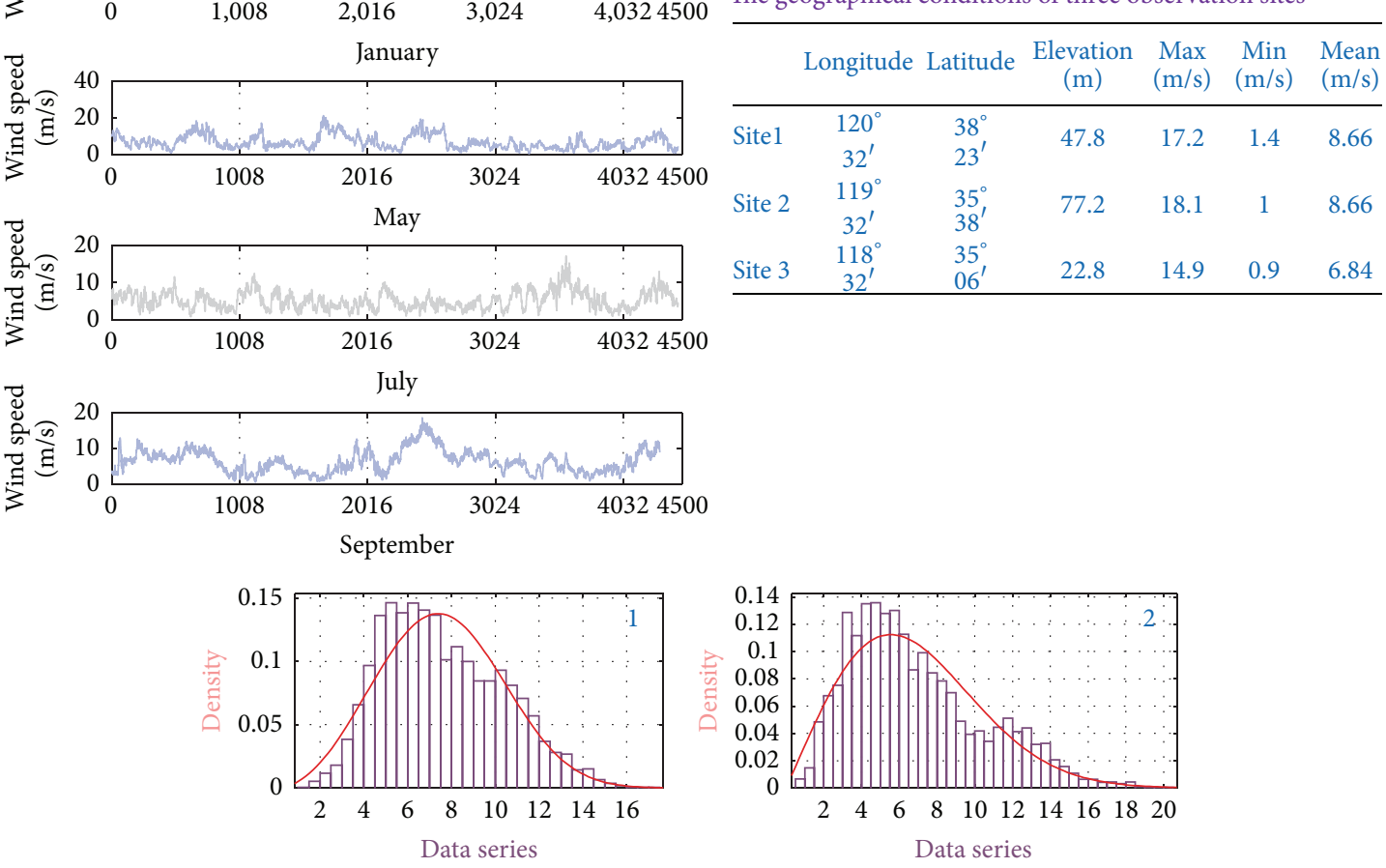

— Data series in January

_ PDF of Weibull

— Data series in May

_ PDF of Weibull
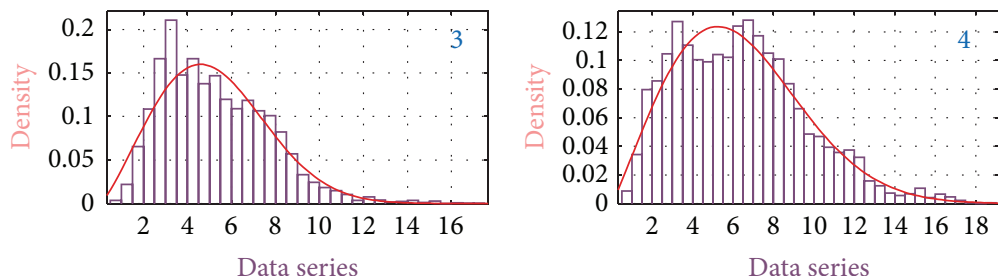

— Data series in July

Data series in September PDF of Weibull

— PDF of Weibull

\begin{tabular}{lcccc}
\hline $\begin{array}{l}\text { Weibull distribution } \\
\text { parameters }\end{array}$ & $\mathrm{a}($ scale) estimate & Std. err. & $\mathrm{b}$ (shape) estimate & Std. err. \\
\hline Data series 1 & 8.47768 & 0.0450284 & 2.97928 & 0.0342044 \\
Data series 2 & 7.73254 & 0.0400057 & 2.03995 & 0.0233793 \\
Data series 3 & 5.90159 & 0.0408818 & 2.29143 & 0.025865 \\
Data series 4 & 7.157 & 0.0448423 & 2.09438 & 0.0247107 \\
\hline
\end{tabular}

FIGURE 1: Original wind speed data collected every 10 minutes in four seasons in 2011 and basic information of these data series. 


\section{Wavelet transform}

Aiming at denoising the original data series on three different observation sites
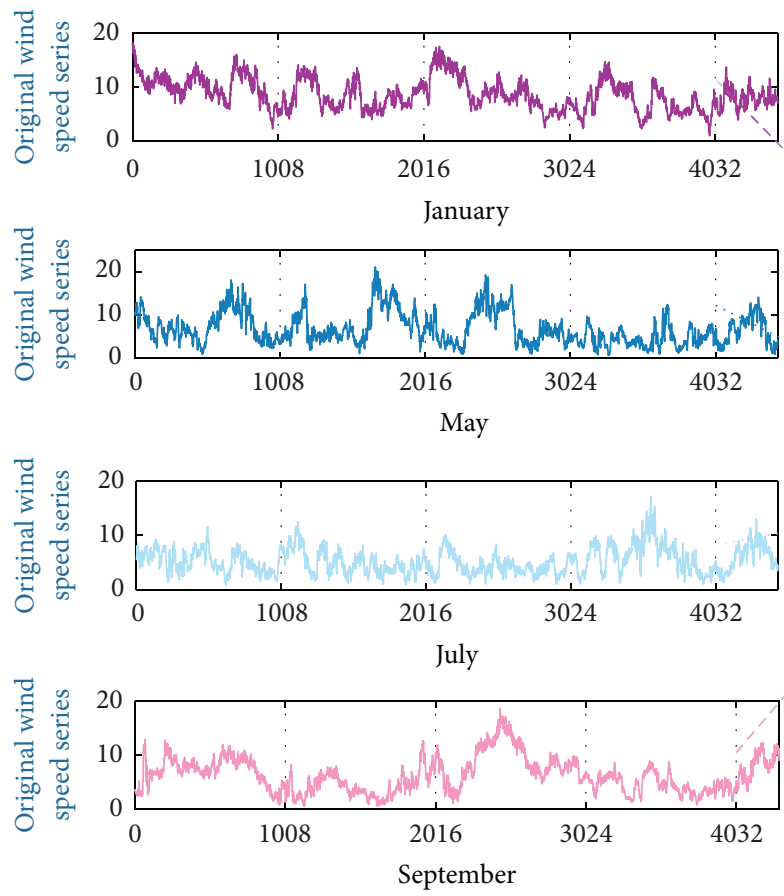
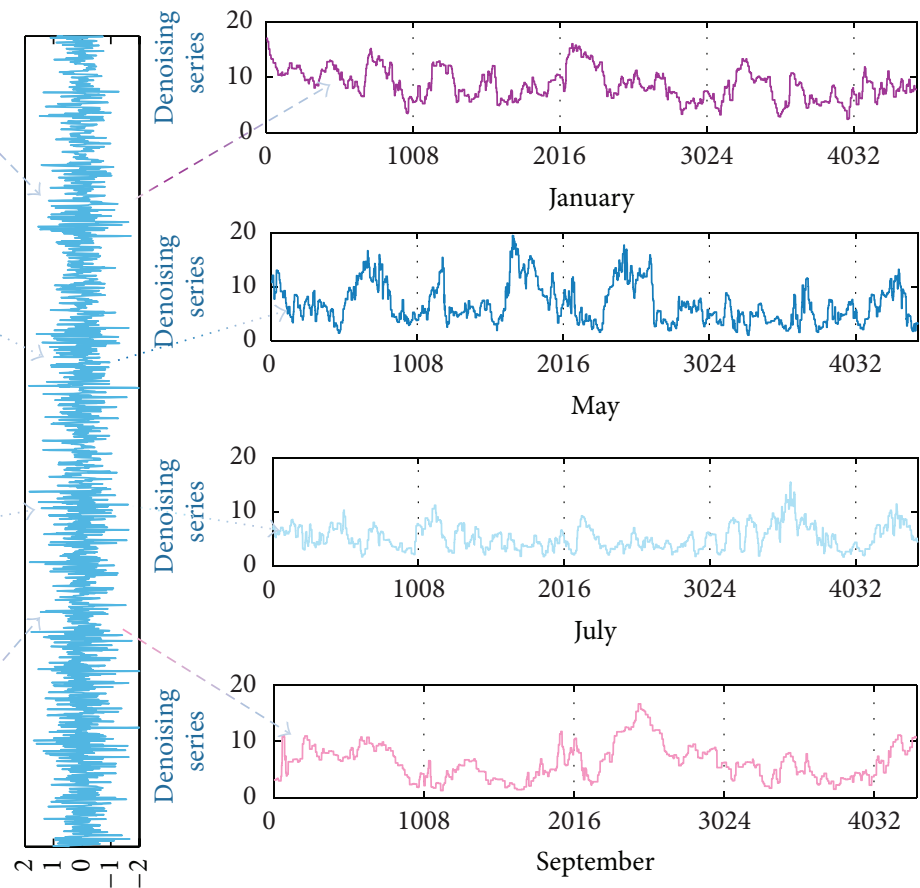

FIGURE 2: Comparison of the original wind speed data series and the results of WT.

the hidden layer, and the output layer [25]. The number of the nodes of the input layer $n$ is determined by our concrete data of wind speed. Herein, our data series is the ten-minute wind speed time series, and three nodes were selected as the input layer $[26,27]$. This choice is based on extensive experiments: the prediction results obtained with three nodes in the input layer are much better than other cases. According to the data series of wind speed, these three input nodes represent the history data at continuous time epochs. Similarly, only one node of the output layer is also used to adapt our data series. As to the hidden layer, it is not difficult to find that it has a great effect on the robustness and stability of the neural network. According to Hecht-Nielsen [28], the proper number of the nodes of the hidden layer, that is, $l=2 n+1$, was selected.

Before implementing the network, it is necessary to normalize the data sets, with both the input and the output data included. Thereafter, the outputs $y_{j}$ of all hidden layer nodes are calculated as

$$
\begin{aligned}
y_{j}=f\left(\sum_{i} w_{i j} x_{i}+b_{j}\right), & \\
& (i=1, \ldots, n ; j=1, \ldots, 2 n+1),
\end{aligned}
$$

where $w_{i j}$ is the weight connected from input nodes $i$ to hidden nodes $j$, while the bias of neural is $b_{j}, f$ is the sigmoid function, and $x_{i}$ represents the value of each input node. Then, calculate the output $W_{1}$ of neural network:

$$
W_{1}=f_{0}\left(\sum_{j} w_{j} y_{j}+b_{0}\right), \quad(j=1, \ldots, 2 n+1),
$$

where $w_{j}$ is the connection weight from hidden layer to output layer, $b_{0}$ is the corresponding bias of neural cell, and the activation function is $f_{0}$. At last, minimize the error via the training process. In this paper, the network was trained firstly; then, the network was validated, and finally we performed model testing. To see more details of the BPANN, [29] can be referred to.

3.3. Artificial Fish Swarm Algorithm (AFSA). Artificial fish swarm algorithm is an effective optimal algorithm for increasing the exploitation capability during the process of searching. As we all know, fish can easily find the area with more nutrition through individual searching or following after the ambient fishes. Li et al. [30] introduced this efficient algorithm in 2002, which models the behaviours of fishes to get the global optimal solution.

The state of the artificial fish can be described as $X=$ $\left(x_{1}, x_{2}, \ldots, x_{n}\right)$, and $x_{i}(i=1,2, \ldots, n)$ represents the initial variable. As the objective function, $Y=f(X)$ is the consistency of the current position where the fish is in. $d_{i, j}=$ $\left\|x_{i}-x_{j}\right\|$ represents the distance of individual fish. Visual is the cognitive distance, while Step and $\delta$ represents the maximum 


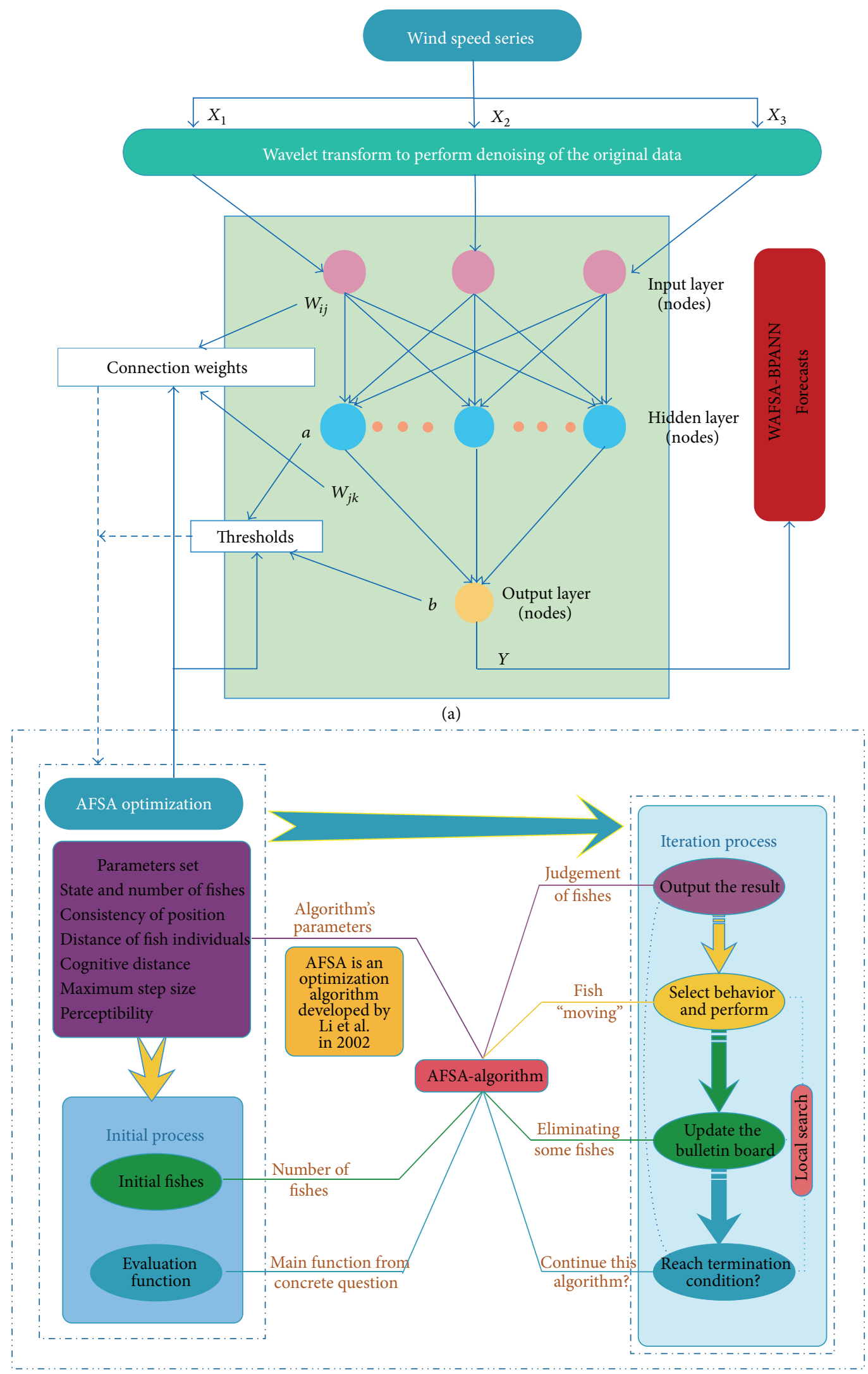

(b)

FIGURE 3: The structure of the hybrid WAFSA-BPANN model. 
step size and factor of congestion degree, respectively. There are four types of behaviours in the AFSA:

(1) Foraging behaviour: the current status of an artificial fish is $X_{i}$, and the fish selects a state $X_{j}$ in its sensing range randomly; if the problem is approximately maximum and $Y_{i}<Y_{j}$ (or $Y_{i}>Y_{j}$ if the problem is approximately minimum; because problems that are approximately maximum or minimum can be converted to each other; in the following, we only discuss the problem that is approximately maximum), then make a step forward in this direction; otherwise, randomly select state $X_{j}$ again and determine whether it meets the condition to move forward; after attempting for try_number times, if the condition still cannot be met, then choose a direction randomly to move a step:

$$
\begin{aligned}
& X_{j}=X_{i}+(2 \operatorname{rand}()-1) \text { Visual }, \\
& X_{i}=X_{i}+(2 \operatorname{rand}()-1) \text { Step. }
\end{aligned}
$$

(2) Swarming behaviour: $X_{i}$ represents the current position of an artificial fish; the task is to explore the numbers of partners $n_{f}$ and the central location $X_{c}$ in the neighbourhood of the current position. If $Y_{c} / n_{f}>$ $\delta y_{i}$, which indicates that there is more nutrition at the centre of partners and less competitiveness, then make a step forward towards the direction of the central of partner; otherwise, execute foraging behaviour:

$$
X_{i}=X_{i-\operatorname{rand}}() \cdot \text { Step } \cdot \frac{X_{c}-X_{i}}{\left\|X_{c}-X_{i}\right\|} .
$$

(3) Following behaviour: the current status of the artificial fish is $X_{i}$; explore the number of partners $Y_{j}$ and the biggest partner $X_{j}$ in the neighbourhood of the current position. If $Y_{c} / n_{f}>\delta y_{i}$, which indicates that the surroundings of $X_{j}$ have more nutrition and are not so congested, then make a step forward towards the direction of $X_{j}$; otherwise, execute foraging behaviour:

$$
X_{i}=X_{i}+\operatorname{rand}() \cdot \text { Step } \cdot \frac{X_{g \text { best }}-X_{i}}{\left\|X_{g \text { best }}-X_{i}\right\|} \text {. }
$$

(4) Random behaviour: implementation of random behaviour is relatively simple; the action is to move a step in a direction that is randomly picked in the field. In fact, this is a default behaviour during foraging behaviour.

There is a bulletin board in the algorithm, which is also defined as an artificial fish, to record the information of the optimal individual [31]. Each of the artificial fish would compare its current state with the state recorded in the bulletin board after every movement, and then substitute it if the current state of the individual is superior to the one recorded in bulletin board before.
According to the nature of the problem to be solved, evaluate the current environment of the fish and then choose a corresponding behaviour. A common method is to choose a behaviour from the alternative behaviours that can make the most progress toward the optimal direction; if there is no choice that can make the next state better than the current state, then the fish has to take a random action.

\section{The Structure of Hybrid WAFSA-BPANN Model}

Just as in the aforementioned references, the initial data of wind speed usually contain much interference, which can affect the accuracy of forecasting; it is therefore a critical procedure for optimal models to select valuable information while filtering the disturbance. Therefore, a relatively mature WT model was introduced to preprocess the original data series, which is nonstationary wind speed data. The sublayers wind speed is a much more stable and smooth time series. Meanwhile, because the initial weights and thresholds of back-propagation artificial neural networks are initialized randomly, it is difficult to reach the best state in most cases. Therefore, finding the best initial parameters of the initial network is greatly needed [32]. Herein, the artificial fish swarm algorithm was applied to finish this work. Based on the WAFSA-BPANN model, the steps of training are depicted as follows.

Step 1. Given the structure of the back-propagation artificial neural network, there are three layers in the network, and the number of nodes is $I, J, K$ in the input layer, the hidden layer, and the output layer, respectively [33].

Step 2. Set the dimensions of the artificial fish, including the weights and thresholds of the artificial neural network:

$$
\begin{aligned}
X & =X\left(v_{11}, \ldots, v_{I 1}, \mu_{1}, \ldots, v_{1 J}, \ldots, v_{I J}, \mu_{J}, \ldots, w_{1 K}, \ldots,\right. \\
& \left.w_{J K}, \theta_{K}\right) .
\end{aligned}
$$

Step 3. Initialize the basic parameters of the artificial fish swarm algorithm, such as the size of the population pop_size, the cognitive distance Visual, the maximum step size Step, and the factor of congestion degree $\delta$, while Max_Gen represents the maximum number of iterations and $\varepsilon$ the objective value.

Step 4. Initialize the iteration step Gen $=0$. Produce artificial fish individuals with a population of pop_size randomly in the feasible region as the initial fish; meanwhile, the component of each individual fish should strictly be random, with digits between $(-1,1)$.

Step 5. Calculate the food concentration $Y$ of the current position of each individual fish, choose the best one, and mark it on the bulletin board.

Step 6. Simulate each individual fish to execute the following behaviour and swarming behaviour, and then calculate $Y$; after comparing and determining the bigger $Y$, execute 


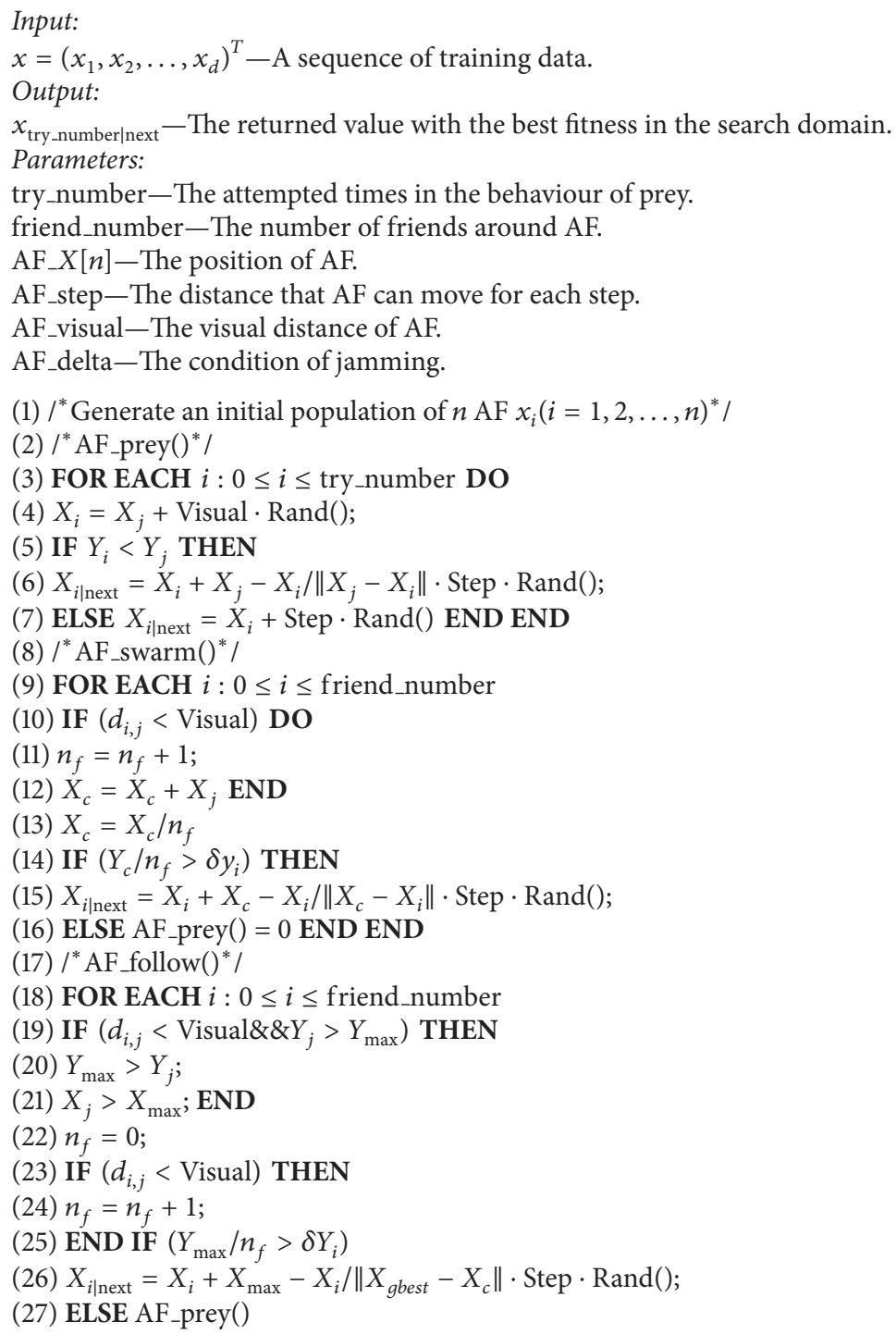

Algorithm 1: AFSA.

the corresponding behaviour (the default action is to execute the foraging behaviour).

Step 7. Update the bulletin board after each iteration, and always substitute the exiting $Y$ with more optimal $Y$ in fish individuals.

Step 8. Judge if the iteration times Gen have already reached Max_Gen or if the error $\varepsilon$ has met the requirement, and output the digit $Y$ on the bulletin board once the termination condition is met; otherwise, make Gen $=\mathrm{Gen}+1$ and go back to Step 6.

The pseudocode of the artificial fish swarm algorithm is listed in Algorithm 1.

Specifics parameters of the AFSA algorithm are presented in Table 1.
TABLE 1: The value of the parameters of AFSA adopted in this paper.

\begin{tabular}{lc}
\hline Parameters & Value \\
\hline Population size & 40 \\
Maximum iterations & 1000 \\
Visual of each individual & 0.5 \\
Step of each fish & 0.01 \\
Try_number of each fish & 10 \\
Congestion degree & 11 \\
Objective value & 0.0001 \\
Time complexity & $O\left(10^{6}\right)$ \\
Space complexity & $O(10)$ \\
\hline
\end{tabular}

The hybrid method has better performance than the traditional back-propagation artificial neural network and other single models, and the structure of the WAFSA-BPANN 
is briefly shown in Figure 3, from which a visualized understanding of the hybrid optimum model can be got.

\section{Simulation and Analysis}

By means of the aforementioned hybrid model and the time series wind speed data, a series of simulations and forecasting have been operated using Matlab R2012b, and the results are discussed as follows.

5.1. Related Index. To ensure fairness and real forecasting results, the data volume, training data set, and forecasting data set are kept unchanged. Herein, three common indexes of error, such as the mean square error (MSE), mean absolute percentage error (MAPE), and the mean absolute error (MAE), are adopted to evaluate the performance of related models, and their specific formulations are shown below:

$$
\begin{aligned}
\text { MAPE } & =\frac{1}{M} \sum_{k=1}^{M}\left|\frac{\widehat{x}_{k}-x_{k}}{x_{k}}\right|, \\
\mathrm{MAE} & =\frac{1}{M} \sum_{k=1}^{M}\left|\widehat{x}_{k}-x_{k}\right|, \\
\mathrm{MSE} & =\frac{1}{M} \sum_{k=1}^{M}\left(\widehat{x}_{k}-x_{k}\right)^{2},
\end{aligned}
$$

where $\widehat{x}_{k}(k=1,2, \ldots, M)$ represents the concrete value of forecasting series and $M$ is the length of the series, while the corresponding value of original data series is $x_{k}$.

\subsection{Discussion of the Results from Different Seasons. Affected} by many meteorological factors which are usually erratic, wind speed fluctuates all of the time, making it difficult to forecast. Based on a previous study, a hybrid new model is proposed in this paper. To achieve intuitive results of the hybrid model, the specific prediction data with a certain time interval on the first weeks of February, June, August, and October are collected, while the training data sets span the whole month of January, May, July, and September, respectively. Thus, the length of the prediction series is 1008 because of the time intervals, which makes it improper to list all these data under the limited space. In this case, forecasting data from several comparative models are recorded every 6 hours. The results from our hybrid model are compared with those from other traditional hybrid models. In this experiment, preprocessed data from four months in different seasons are utilized to train the neural network, and upon training the neural network, the final forecasting is made. Herein, data collected from January 1 to January 31 are utilized as the training set to train the network, upon which the prediction of wind speed from February 1 to February 7 is achieved. Next, preprocessed data acquired from May 1 to May 31 are used to train the artificial neural network, and the data from June 1 to June 7 are the relevant testing set. According to the same principle, the remaining training data series are formed. For example, the testing data from August 1 to August 7 are obtained from the neural network, which are trained by wind speed data spanning July 1 to July 31 .

Tables 2, 3, 4, and 5 display the prediction results of four seasons, respectively. To be specific, forecasting results in the first week of February obtained from different models are presented in Table 2. Similarly, Tables 3, 4, and 5 represent the forecasting results in other three seasons.

As is depicted in Tables 2, 3, 4, and 5 and Figure 4, the specific forecasting results are enumerated vividly based on four different models: the initial back-propagation artificial neural network, the ARIMA model, the ARIMA with data from wavelet transforming (WT-ARIMA), and the hybrid BP network with optimal parameters as well as the preprocessed data. Through the comparison of data, it can be found that the MAPE of WAFSA-BPANN is relatively more smooth and smaller than the other three models. Here, we can know that the wind speed in February has a remarkable fluctuation, which results in a relatively big error in all four models. Through further analysis, a rough speculation can be formed: compared with other times, the falling of temperature in the winter may lead to the fluctuation of the wind speed.

\subsection{Discussion of the Results from Different Observation} Sites. To test the stability of the experiments as well as the effectiveness of the proposed hybrid model, the scope of the training data and testing data was enlarged, including four whole months in different seasons. At the same time, horizontal comparison is also employed to strengthen the validity of the proposed hybrid models. Furthermore, to eliminate random error in wind speed data, data fields from three different observation sites are applied in the process of simulation and forecasting. All these efforts aim to reduce the randomness of the experiments as well as testify the effectiveness of the proposed models, and the analysis results of data series gathered from three different observation sites are presented in Tables 6-9.

Obvious improvements in the performance of the hybrid models can be noticed, which can be read from all three error criteria. And the superiority of our WAFSA-BPANN model exists in observation site 1 , site 2 , and site 3 . As is depicted in Table 6 , it is not hard to find that there are $1.28 \%, 1.6 \%, 1.31 \%$, $1.18 \%$, and $1.13 \%$ reductions in MAPE, $0.1419,0.1586,0.1447$, 0.1113 , and 0.1199 reductions in MSE, and 0.0871, 0.1048, $0.0888,0.073$, and 0.0779 reductions in MAE, respectively, of the hybrid model proposed in this paper when compared with BPANN, ARIMA, AFSA-BPANN, WT-BPANN, and WT-ARIMA model.

On other aspects, through the horizontal comparison of the data shown in Tables 6, 7, 8, and 9, WT's superior performance on the three error indexes, compared with the performances of the other traditional models, can be seen. The average index of errors in all tables covering four seasons also indicates this rule: the hybrid model indeed has much more superiority when dealing with the original data set, which contains much noise and fluctuation. In other words, the process of denoising of WT has a critical effect on the final forecasting when analysing the comprehensive comparison, from which we can find an obvious improvement for 


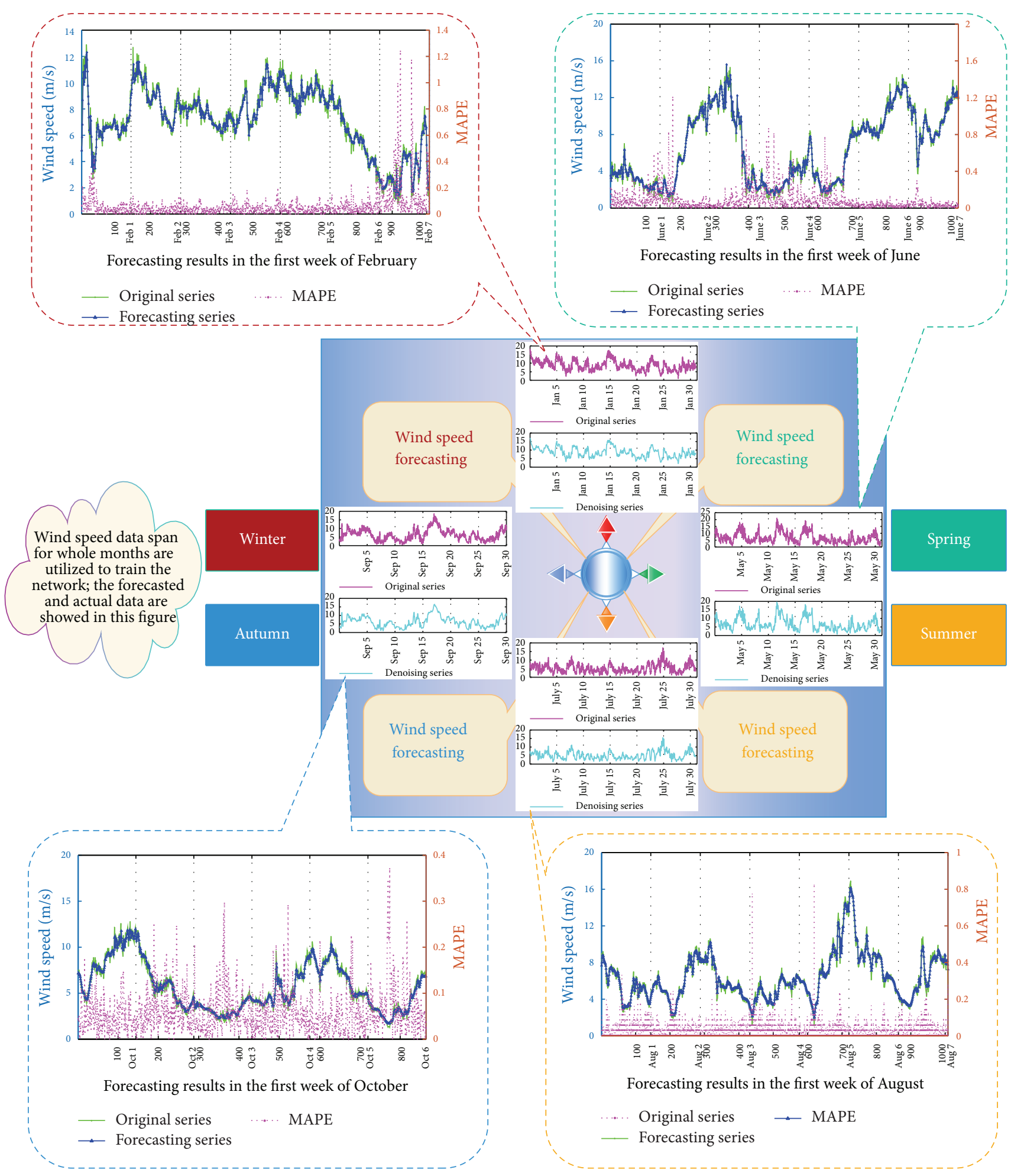

FIGURE 4: The specific results of different models.

the model when the wavelet transform is added. Synthesizing these diagrams, a rough rule can be observed, showing that the wavelet transform has an impact on simulation and forecasting: the tendency of the error index is more flat compared with the original error. The hybrid model (WAFSA-BPANN) can at least be confirmed to finish forecasting the wind speed with a tolerated error.

Moreover, it is necessary to consider the running time when implementing these models. On average, the BPANN model is the fastest which usually costs less than two seconds, 
TABLE 2: The forecasting results of February from observation site 1 on a given time interval.

\begin{tabular}{|c|c|c|c|c|c|c|c|c|c|c|}
\hline \multirow{2}{*}{$\begin{array}{l}\text { Date } \\
\text { February }\end{array}$} & \multirow{2}{*}{ Time (h) } & \multirow{2}{*}{$\begin{array}{c}\text { Actual } \\
\text { value }\end{array}$} & \multicolumn{2}{|c|}{ BPANN } & \multicolumn{2}{|c|}{ ARIMA } & \multicolumn{2}{|c|}{ WT-ARIMA } & \multicolumn{2}{|c|}{ WAFSA-BPANN } \\
\hline & & & Forecasting & MAPE (\%) & Forecasting & MAPE (\%) & Forecasting & MAPE (\%) & Forecasting & MAPE (\%) \\
\hline \multirow{4}{*}{ Feb. 1} & 0:00 & 7.9 & 7.025 & 11.071 & 7.080 & 10.378 & 7.759 & 1.779 & 7.681 & 2.778 \\
\hline & $6: 00$ & 7.4 & 7.082 & 4.292 & 7.085 & 4.253 & 7.140 & 3.513 & 7.171 & 3.094 \\
\hline & $12: 00$ & 3.1 & 3.123 & 0.743 & 3.014 & 2.782 & 2.981 & 3.832 & 3.158 & 1.880 \\
\hline & $18: 00$ & 4.4 & 4.385 & 0.339 & 4.287 & 2.571 & 4.268 & 3.003 & 4.404 & 0.102 \\
\hline \multirow{4}{*}{ Feb. 2} & $0: 00$ & 5 & 4.619 & 7.614 & 4.320 & 13.609 & 4.500 & 9.998 & 4.370 & 12.597 \\
\hline & $6: 00$ & 4.7 & 4.877 & 3.760 & 4.789 & 1.900 & 4.767 & 1.429 & 4.844 & 3.069 \\
\hline & $12: 00$ & 2.8 & 2.825 & 0.899 & 2.492 & 10.995 & 2.570 & 8.218 & 2.823 & 0.838 \\
\hline & $18: 00$ & 8.2 & 7.712 & 5.946 & 7.623 & 7.033 & 7.859 & 4.158 & 7.859 & 4.153 \\
\hline \multirow{4}{*}{ Feb. 3} & $0: 00$ & 8.3 & 8.179 & 1.461 & 8.180 & 1.443 & 8.221 & 0.947 & 8.478 & 2.150 \\
\hline & $6: 00$ & 8.4 & 8.388 & 0.146 & 8.253 & 1.746 & 8.442 & 0.505 & 8.520 & 1.428 \\
\hline & $12: 00$ & 4.6 & 5.234 & 13.778 & 5.172 & 12.440 & 5.239 & 13.895 & 5.224 & 13.568 \\
\hline & $18: 00$ & 5.4 & 5.167 & 4.314 & 5.139 & 4.829 & 5.107 & 5.431 & 5.375 & 0.469 \\
\hline \multirow{4}{*}{ Feb. 4} & $0: 00$ & 3 & 3.048 & 1.616 & 2.948 & 1.728 & 2.867 & 4.435 & 3.056 & 1.869 \\
\hline & $6: 00$ & 4.7 & 4.553 & 3.126 & 4.692 & 0.165 & 4.383 & 6.750 & 4.831 & 2.783 \\
\hline & $12: 00$ & 5.6 & 5.046 & 9.896 & 4.412 & 21.211 & 4.785 & 14.558 & 4.639 & 17.166 \\
\hline & $18: 00$ & 5.6 & 5.650 & 0.888 & 5.596 & 0.079 & 5.583 & 0.304 & 5.519 & 1.452 \\
\hline \multirow{4}{*}{ Feb. 5} & $0: 00$ & 6 & 6.075 & 1.255 & 6.073 & 1.214 & 5.960 & 0.669 & 6.196 & 3.264 \\
\hline & $6: 00$ & 3 & 3.325 & 10.835 & 3.256 & 8.517 & 3.176 & 5.874 & 3.274 & 9.138 \\
\hline & $12: 00$ & 6.3 & 6.925 & 9.914 & 6.936 & 10.096 & 6.875 & 9.130 & 6.701 & 6.360 \\
\hline & 18:00 & 7.5 & 8.285 & 10.467 & 8.300 & 10.672 & 8.118 & 8.236 & 8.306 & 10.742 \\
\hline \multirow{4}{*}{ Feb. 6} & $0: 00$ & 15 & 13.412 & 10.590 & 13.409 & 10.606 & 13.222 & 11.850 & 14.511 & 3.263 \\
\hline & $6: 00$ & 9.4 & 9.374 & 0.274 & 9.309 & 0.965 & 9.445 & 0.475 & 9.136 & 2.809 \\
\hline & $12: 00$ & 8.6 & 9.010 & 4.771 & 8.956 & 4.136 & 9.058 & 5.323 & 8.938 & 3.933 \\
\hline & 18:00 & 6.5 & 6.402 & 1.502 & 6.300 & 3.073 & 6.421 & 1.214 & 6.601 & 1.556 \\
\hline \multirow{4}{*}{ Feb. 7} & $0: 00$ & 4.6 & 4.679 & 1.721 & 4.659 & 1.278 & 4.601 & 0.023 & 4.502 & 2.135 \\
\hline & $6: 00$ & 3.4 & 3.716 & 9.308 & 3.488 & 2.591 & 3.610 & 6.177 & 3.556 & 4.583 \\
\hline & $12: 00$ & 8.7 & 10.145 & 16.614 & 10.084 & 15.904 & 10.176 & 16.970 & 9.207 & 5.830 \\
\hline & $18: 00$ & 7.9 & 8.217 & 4.010 & 8.219 & 4.044 & 7.995 & 1.196 & 8.179 & 3.535 \\
\hline
\end{tabular}

and this can be seen in Tables 6, 7,8, and 9. It is still very quick even the process of wavelet transform is added. The ARIMA and WT-ARIMA model are relatively fast with the running time about forty seconds. However, the speed of model always slows down when it involves the AFSA algorithm, which usually lasts 150 seconds. Through further analysis, it is not hard to explain the longer period of hybrid model proposed in this paper since there are so many loops and iterations in the AFSA algorithm.

Though much more time is needed when applying the hybrid model, it is still a reasonable choice to make wind speed forecasting for wind farms, for the improvement of prediction accuracy can compensate the costs in time and bring more profits both in technology and economy.

5.4. Comprehensive Comparison between Different Models. Table 10 shows the final comprehensive comparison between the traditional and hybrid models. Based on the metrics tabularized in Table 10, the overall perspective of each model can be seen distinctly. It should be noted here that all of those digits are the means of the whole experiment. For instance, the MAE of the BPANN model is 0.4284, which is the mean of the aforementioned forecasting results from these three observations sites covering four seasons. The MSE and MAPE are formed in the similar way.

It can be found that, when compared with BPANN, ARIMA, AFSA-BPANN, WT-BPANN, and WT-ARIMA, there are $24.7 \%, 25.5 \%, 24.78 \%, 24.64 \%$, and $25.53 \%$ improvements in MAE, $45.25 \%, 46.2 \%, 45.01 \%, 45.58 \%$, and $46.93 \%$ improvements in MSE, and 21.28\%, 21.17\%, 21.87\%, 20.1\%, and $20.52 \%$ improvements in MAPE, respectively, of the hybrid model proposed in this paper. Similarly, reductions in MAPE, MSE, and MAE occurred at all three observation sites in four seasons which are presented in aforementioned tables.

Through the aforementioned table and analysis, a general rule could be summarised now: the proposed WAFSABPANN can always perform better than other referred models, which is obviously embodied in MAPE, MAE, and MSE. Above all, a visualized conclusion can be reached: the proposed hybrid model performs better than the others. In summary, the proposed optimal hybrid model can, to some extent, make relatively better forecasts than the traditional prediction models. In other words, the optimal algorithm introduced in this paper is suitable to be applied to optimizing the traditional back-propagation artificial neural network based on the wind speed data. 
TABLE 3: The forecasting results of June from observation site 1 on a given time interval.

\begin{tabular}{|c|c|c|c|c|c|c|c|c|c|c|}
\hline \multirow{2}{*}{$\begin{array}{l}\text { Date } \\
\text { June }\end{array}$} & \multirow{2}{*}{ Time $(\mathrm{h})$} & \multirow{2}{*}{$\begin{array}{c}\text { Actual } \\
\text { value }\end{array}$} & \multicolumn{2}{|c|}{ BPANN } & \multicolumn{2}{|c|}{ ARIMA } & \multicolumn{2}{|c|}{ WT-ARIMA } & \multicolumn{2}{|c|}{ WAFSA-BPANN } \\
\hline & & & Forecasting & MAPE (\%) & Forecasting & MAPE (\%) & Forecasting & MAPE (\%) & Forecasting & MAPE (\%) \\
\hline \multirow{4}{*}{ June. 1} & $0: 00$ & 2.9 & 4.423 & 52.508 & 4.293 & 48.044 & 3.292 & 13.530 & 3.529 & 21.693 \\
\hline & $6: 00$ & 3.7 & 2.748 & 25.728 & 2.653 & 28.287 & 2.540 & 31.358 & 3.387 & 8.449 \\
\hline & $12: 00$ & 2.6 & 3.235 & 24.425 & 3.144 & 20.929 & 3.126 & 20.230 & 2.991 & 15.027 \\
\hline & 18:00 & 1.8 & 2.309 & 28.265 & 2.138 & 18.759 & 2.078 & 15.423 & 2.151 & 19.499 \\
\hline \multirow{4}{*}{ June. 2} & $0: 00$ & 1.6 & 1.690 & 5.632 & 1.464 & 8.529 & 1.385 & 13.407 & 1.730 & 8.116 \\
\hline & $6: 00$ & 0.7 & 1.389 & 98.435 & 1.091 & 55.881 & 1.105 & 57.881 & 1.543 & 120.427 \\
\hline & $12: 00$ & 6.1 & 6.150 & 0.817 & 6.133 & 0.537 & 6.247 & 2.403 & 6.261 & 2.636 \\
\hline & 18:00 & 9.5 & 9.949 & 4.728 & 9.949 & 4.726 & 10.032 & 5.595 & 9.857 & 3.758 \\
\hline \multirow{4}{*}{ June. 3} & $0: 00$ & 10.8 & 10.788 & 0.112 & 10.685 & 1.063 & 10.748 & 0.481 & 10.982 & 1.684 \\
\hline & $6: 00$ & 12 & 12.724 & 6.034 & 12.742 & 6.183 & 12.790 & 6.581 & 12.276 & 2.300 \\
\hline & $12: 00$ & 8.9 & 9.151 & 2.815 & 9.332 & 4.859 & 9.199 & 3.357 & 9.304 & 4.542 \\
\hline & 18:00 & 2.4 & 1.813 & 24.455 & 1.785 & 25.627 & 1.631 & 32.040 & 2.172 & 9.482 \\
\hline \multirow{4}{*}{ June. 4} & $0: 00$ & 2.4 & 2.376 & 0.983 & 2.200 & 8.338 & 2.207 & 8.022 & 2.340 & 2.509 \\
\hline & $6: 00$ & 1.9 & 2.143 & 12.795 & 1.901 & 0.049 & 1.900 & 0.024 & 1.922 & 1.150 \\
\hline & $12: 00$ & 2.9 & 3.028 & 4.404 & 2.844 & 1.943 & 2.915 & 0.504 & 2.872 & 0.970 \\
\hline & 18:00 & 5.4 & 4.435 & 17.873 & 4.395 & 18.611 & 4.410 & 18.337 & 4.398 & 18.551 \\
\hline \multirow{4}{*}{ June. 5} & $0: 00$ & 8 & 7.444 & 6.956 & 7.369 & 7.886 & 7.566 & 5.429 & 7.321 & 8.491 \\
\hline & $6: 00$ & 1.3 & 1.570 & 20.753 & 1.400 & 7.701 & 1.269 & 2.365 & 1.763 & 35.644 \\
\hline & $12: 00$ & 2.2 & 2.652 & 20.543 & 2.482 & 12.801 & 2.487 & 13.066 & 2.665 & 21.118 \\
\hline & 18:00 & 5.9 & 5.879 & 0.351 & 5.781 & 2.015 & 5.895 & 0.081 & 5.704 & 3.321 \\
\hline \multirow{4}{*}{ June. 6} & $0: 00$ & 7.8 & 7.861 & 0.776 & 7.841 & 0.525 & 7.884 & 1.081 & 8.072 & 3.482 \\
\hline & $6: 00$ & 8.3 & 8.286 & 0.167 & 8.312 & 0.150 & 8.277 & 0.283 & 8.431 & 1.581 \\
\hline & $12: 00$ & 9.5 & 8.627 & 9.190 & 8.635 & 9.106 & 8.702 & 8.398 & 9.268 & 2.443 \\
\hline & 18:00 & 14.2 & 12.340 & 13.098 & 12.331 & 13.165 & 12.412 & 12.593 & 12.911 & 9.078 \\
\hline \multirow{4}{*}{ June. 7} & $0: 00$ & 11.4 & 10.582 & 7.179 & 10.742 & 5.775 & 10.578 & 7.214 & 11.174 & 1.982 \\
\hline & $6: 00$ & 7 & 6.839 & 2.302 & 6.859 & 2.014 & 6.914 & 1.229 & 6.988 & 0.175 \\
\hline & $12: 00$ & 7.3 & 6.744 & 7.614 & 6.845 & 6.235 & 6.794 & 6.938 & 7.374 & 1.013 \\
\hline & $18: 00$ & 10.3 & 10.616 & 3.068 & 10.546 & 2.388 & 10.748 & 4.348 & 10.445 & 1.404 \\
\hline
\end{tabular}

\section{Conclusions}

With the ongoing need for wind power, accurate wind speed forecasting has a strong impact on wind farm management, even on the entire wind power dispatch hybrid system. Therefore, a new hybrid model is proposed in this paper to solve this tough task. Firstly, based on WT, extra noise in the original wind speed data series which are nonstationary is eliminated while the remaining data contain most of the effective information, which is critical for forecasting wind speed. Next, to achieve the most proper parameters of the back-propagation artificial neural network, AFSA, as an effective method, is applied in this paper, and with the optimal parameters, the initial network is formed. Then, the preprocessed wind speed data are utilized to train the BPANN artificial neural network; in the final process, the forecasting of wind speed is obtained from the trained network. A number of sets of wind speed data from four different observation sites are gathered to execute the simulation and forecasting. Meanwhile, three error metrics are built up to evaluate the performance of the models. Through comprehensive comparison, the conclusion elicited is that the proposed intelligent hybrid model (WAFSA-BPANN) outperforms the other traditional models, such as BPANN, ARIMA, AFSA-BPANN,
WT-BPANN, and WT-ARIMA. Hence, WAFSA-BPANN is an effective and reliable model for short-term wind speed forecasting in wind farms of China.

Though relatively superior precision of wind speed prediction can be obtained applying the hybrid model proposed in this paper, there is still room for improvements. Investigating these forecasting approaches, such as the combination of numerical simulation and intelligence algorithms, could be a future research topic.

\section{Abbreviations}

AFSA: Artificial fish swarm algorithm

ANN: Artificial neural network

AR: $\quad$ Auto Regressive Model

ARIMA: Auto Regressive Integrated Moving Average

BEVs: Battery electric vehicles

BMS: $\quad$ Battery management system

BPANN: Back-propagation artificial neural network

ESM: Exponential Smoothing Method

EVs: $\quad$ Electric vehicles

$\mathrm{GM}(1, n)$ : Grey prediction model

KF: Kalman filter 
TABLE 4: The forecasting results of August from observation site 1 on a given time interval.

\begin{tabular}{|c|c|c|c|c|c|c|c|c|c|c|}
\hline \multirow{2}{*}{$\begin{array}{l}\text { Date } \\
\text { August }\end{array}$} & \multirow{2}{*}{ Time (h) } & \multirow{2}{*}{$\begin{array}{c}\text { Actual } \\
\text { value }\end{array}$} & \multicolumn{2}{|c|}{ BPANN } & \multicolumn{2}{|c|}{ ARIMA } & \multicolumn{2}{|c|}{ WT-ARIMA } & \multicolumn{2}{|c|}{ WAFSA-BPANN } \\
\hline & & & Forecasting & MAPE (\%) & Forecasting & MAPE (\%) & Forecasting & MAPE (\%) & Forecasting & MAPE (\%) \\
\hline \multirow{4}{*}{ Aug. 1} & $0: 00$ & 4.8 & 4.539 & 5.448 & 4.595 & 4.263 & 4.403 & 8.263 & 4.639 & 3.356 \\
\hline & $6: 00$ & 4.1 & 4.536 & 10.642 & 4.529 & 10.454 & 4.442 & 8.341 & 3.897 & 4.951 \\
\hline & $12: 00$ & 2.4 & 2.586 & 7.731 & 2.434 & 1.437 & 2.493 & 3.869 & 2.579 & 7.472 \\
\hline & 18:00 & 1.7 & 1.751 & 3.013 & 1.566 & 7.872 & 1.510 & 11.184 & 1.838 & 8.139 \\
\hline \multirow{4}{*}{ Aug. 2} & $0: 00$ & 3.5 & 3.865 & 10.423 & 3.787 & 8.201 & 3.775 & 7.845 & 3.848 & 9.951 \\
\hline & $6: 00$ & 3 & 2.971 & 0.959 & 2.850 & 4.986 & 2.816 & 6.137 & 2.838 & 5.413 \\
\hline & $12: 00$ & 2.4 & 2.912 & 21.340 & 2.796 & 16.501 & 2.835 & 18.126 & 2.642 & 10.068 \\
\hline & 18:00 & 3.3 & 3.421 & 3.669 & 3.323 & 0.702 & 3.302 & 0.049 & 3.352 & 1.585 \\
\hline \multirow{4}{*}{ Aug. 3} & $0: 00$ & 2.8 & 2.853 & 1.900 & 2.816 & 0.583 & 2.682 & 4.230 & 2.875 & 2.676 \\
\hline & $6: 00$ & 5.1 & 4.442 & 12.903 & 4.376 & 14.201 & 4.345 & 14.800 & 4.379 & 14.141 \\
\hline & $12: 00$ & 4.3 & 3.970 & 7.675 & 3.939 & 8.400 & 3.886 & 9.621 & 3.775 & 12.218 \\
\hline & 18:00 & 2 & 2.168 & 8.380 & 2.060 & 2.978 & 2.087 & 4.344 & 2.383 & 19.144 \\
\hline \multirow{4}{*}{ Aug. 4} & $0: 00$ & 2.3 & 1.925 & 16.306 & 1.740 & 24.338 & 1.743 & 24.214 & 2.298 & 0.088 \\
\hline & $6: 00$ & 3 & 3.221 & 7.362 & 3.134 & 4.451 & 3.099 & 3.287 & 3.428 & 14.257 \\
\hline & $12: 00$ & 3.1 & 2.504 & 19.233 & 2.418 & 22.009 & 2.411 & 22.242 & 3.022 & 2.504 \\
\hline & 18:00 & 2.8 & 2.912 & 3.999 & 2.858 & 2.064 & 2.815 & 0.521 & 2.661 & 4.980 \\
\hline \multirow{4}{*}{ Aug. 5} & $0: 00$ & 1.8 & 1.648 & 8.435 & 1.529 & 15.072 & 1.479 & 17.849 & 1.888 & 4.861 \\
\hline & $6: 00$ & 0.8 & 1.581 & 97.609 & 1.474 & 84.254 & 1.398 & 74.703 & 1.514 & 89.304 \\
\hline & $12: 00$ & 1.4 & 1.718 & 22.680 & 1.567 & 11.911 & 1.609 & 14.911 & 1.897 & 35.478 \\
\hline & 18:00 & 4.8 & 4.287 & 10.696 & 4.256 & 11.328 & 4.387 & 8.607 & 4.635 & 3.429 \\
\hline \multirow{4}{*}{ Aug. 6} & $0: 00$ & 1.3 & 1.444 & 11.099 & 1.284 & 1.226 & 1.161 & 10.729 & 1.746 & 34.313 \\
\hline & $6: 00$ & 3.3 & 3.136 & 4.964 & 3.120 & 5.445 & 3.076 & 6.792 & 3.039 & 7.923 \\
\hline & $12: 00$ & 3.9 & 3.719 & 4.651 & 3.689 & 5.399 & 3.600 & 7.684 & 3.472 & 10.980 \\
\hline & 18:00 & 1.9 & 2.598 & 36.716 & 2.455 & 29.233 & 2.511 & 32.145 & 2.490 & 31.040 \\
\hline \multirow{4}{*}{ Aug. 7} & $0: 00$ & 2.6 & 2.796 & 7.526 & 2.764 & 6.291 & 2.782 & 7.007 & 2.787 & 7.203 \\
\hline & $6: 00$ & 3.8 & 3.768 & 0.837 & 3.710 & 2.381 & 3.772 & 0.733 & 3.762 & 0.989 \\
\hline & $12: 00$ & 4.5 & 4.019 & 10.688 & 3.996 & 11.189 & 3.995 & 11.233 & 3.973 & 11.722 \\
\hline & 18:00 & 12.1 & 10.417 & 13.907 & 10.259 & 15.216 & 10.559 & 12.733 & 11.377 & 5.975 \\
\hline
\end{tabular}

TABLE 5: The forecasting results of October from observation site 1 on a given time interval.

\begin{tabular}{|c|c|c|c|c|c|c|c|c|c|c|}
\hline \multirow{2}{*}{$\begin{array}{l}\text { Date } \\
\text { October }\end{array}$} & \multirow{2}{*}{ Time (h) } & \multirow{2}{*}{$\begin{array}{l}\text { Actual } \\
\text { value }\end{array}$} & \multicolumn{2}{|c|}{ BPANN } & \multicolumn{2}{|c|}{ ARIMA } & \multicolumn{2}{|c|}{ WT-ARIMA } & \multicolumn{2}{|c|}{ WAFSA-BPANN } \\
\hline & & & Forecasting & MAPE (\%) & Forecasting & MAPE (\%) & Forecasting & MAPE (\%) & Forecasting & MAPE (\%) \\
\hline \multirow{4}{*}{ Oct. 2} & $0: 00$ & 7.5 & 7.452 & 0.645 & 7.384 & 1.541 & 6.322 & 15.709 & 7.160 & 4.527 \\
\hline & $6: 00$ & 8.5 & 8.185 & 3.706 & 8.129 & 4.368 & 8.368 & 1.548 & 7.955 & 6.406 \\
\hline & $12: 00$ & 9.1 & 9.412 & 3.431 & 9.422 & 3.543 & 9.511 & 4.513 & 9.228 & 1.408 \\
\hline & 18:00 & 10.7 & 11.173 & 4.423 & 10.695 & 0.051 & 10.442 & 2.408 & 10.988 & 2.690 \\
\hline \multirow{4}{*}{ Oct. 3} & $0: 00$ & 10.5 & 11.719 & 11.610 & 11.723 & 11.649 & 11.765 & 12.052 & 10.970 & 4.480 \\
\hline & $6: 00$ & 6.5 & 6.817 & 4.883 & 6.912 & 6.337 & 6.770 & 4.148 & 7.017 & 7.957 \\
\hline & $12: 00$ & 5.7 & 5.273 & 7.492 & 5.344 & 6.247 & 5.082 & 10.841 & 5.796 & 1.683 \\
\hline & 18:00 & 4.4 & 4.188 & 4.818 & 4.166 & 5.316 & 4.199 & 4.571 & 4.207 & 4.394 \\
\hline \multirow{4}{*}{ Oct. 4} & $0: 00$ & 3.8 & 4.424 & 16.428 & 4.477 & 17.817 & 4.434 & 16.675 & 4.179 & 9.983 \\
\hline & $6: 00$ & 3.3 & 3.432 & 3.986 & 3.353 & 1.592 & 3.392 & 2.781 & 3.212 & 2.677 \\
\hline & $12: 00$ & 3.1 & 3.176 & 2.442 & 2.955 & 4.673 & 3.075 & 0.795 & 2.574 & 16.962 \\
\hline & 18:00 & 2.9 & 2.579 & 11.065 & 2.474 & 14.683 & 2.476 & 14.619 & 2.667 & 8.043 \\
\hline \multirow{4}{*}{ Oct. 5} & $0: 00$ & 4.3 & 4.483 & 4.267 & 4.449 & 3.459 & 4.499 & 4.622 & 4.317 & 0.392 \\
\hline & $6: 00$ & 3.8 & 3.921 & 3.187 & 3.918 & 3.114 & 3.894 & 2.481 & 4.034 & 6.146 \\
\hline & $12: 00$ & 6.6 & 6.225 & 5.685 & 6.190 & 6.214 & 6.159 & 6.687 & 6.106 & 7.480 \\
\hline & 18:00 & 7.5 & 6.712 & 10.508 & 6.557 & 12.568 & 6.865 & 8.468 & 7.105 & 5.272 \\
\hline \multirow{4}{*}{ Oct. 6} & $0: 00$ & 9.4 & 8.985 & 4.412 & 8.982 & 4.450 & 9.020 & 4.038 & 9.024 & 4.000 \\
\hline & $6: 00$ & 8.6 & 8.160 & 5.116 & 8.164 & 5.067 & 8.075 & 6.103 & 8.192 & 4.740 \\
\hline & $12: 00$ & 6.8 & 7.209 & 6.008 & 7.286 & 7.140 & 7.216 & 6.119 & 7.328 & 7.765 \\
\hline & 18:00 & 4.2 & 4.300 & 2.391 & 4.402 & 4.814 & 4.275 & 1.794 & 4.621 & 10.013 \\
\hline \multirow{4}{*}{ Oct. 7} & $0: 00$ & 3.9 & 4.456 & 14.266 & 4.519 & 15.864 & 4.371 & 12.067 & 4.354 & 11.645 \\
\hline & $6: 00$ & 2.6 & 2.573 & 1.038 & 2.512 & 3.398 & 2.484 & 4.467 & 2.441 & 6.111 \\
\hline & $12: 00$ & 3.1 & 3.502 & 12.952 & 3.448 & 11.232 & 3.519 & 13.511 & 3.046 & 1.744 \\
\hline & $18: 00$ & 4.6 & 4.641 & 0.884 & 4.675 & 1.624 & 4.632 & 0.689 & 4.670 & 1.522 \\
\hline
\end{tabular}


TABLE 6: Errors of different traditional models in February.

\begin{tabular}{|c|c|c|c|c|c|}
\hline & Models & $\operatorname{MAE}(\mathrm{m} / \mathrm{s})$ & $\operatorname{MSE}\left(\mathrm{m}^{2} / \mathrm{s}^{2}\right)$ & MAPE (\%) & Running time (s) \\
\hline \multirow{6}{*}{$\begin{array}{l}\text { Observation } \\
\text { site } 1\end{array}$} & BPANN & 0.3911 & 0.3041 & 6.68 & 2.8502 \\
\hline & ARIMA & 0.4088 & 0.3208 & 7 & 47.2708 \\
\hline & AFSA-BPANN & 0.3928 & 0.3069 & 6.71 & 105.3652 \\
\hline & WT-BPANN & 0.3770 & 0.2735 & 6.58 & 5.1081 \\
\hline & WT-ARIMA & 0.3819 & 0.2821 & 6.53 & 40.7528 \\
\hline & WAFSA-BPANN & 0.3040 & 0.1622 & 5.4 & 156.3590 \\
\hline \multirow{6}{*}{$\begin{array}{l}\text { Observation } \\
\text { site } 2\end{array}$} & BPANN & 0.4219 & 0.3304 & 9.5 & 2.0659 \\
\hline & ARIMA & 0.4415 & 0.3526 & 9.94 & 35.5574 \\
\hline & AFSA-BPANN & 0.4307 & 0.3389 & 9.78 & 106.2827 \\
\hline & WT-BPANN & 0.4087 & 0.3045 & 9 & 2.4668 \\
\hline & WT-ARIMA & 0.4130 & 0.3109 & 9.03 & 42.0537 \\
\hline & WAFSA-BPANN & 0.3134 & 0.1674 & 7.44 & 148.8582 \\
\hline \multirow{6}{*}{$\begin{array}{l}\text { Observation } \\
\text { site } 3\end{array}$} & BPANN & 0.3975 & 0.2845 & 8.27 & 0.5424 \\
\hline & ARIMA & 0.4098 & 0.3021 & 8.43 & 42.4113 \\
\hline & AFSA-BPANN & 0.3949 & 0.2793 & 8.24 & 105.7024 \\
\hline & WT-BPANN & 0.3833 & 0.2654 & 8.05 & 0.7321 \\
\hline & WT-ARIMA & 0.3849 & 0.2694 & 7.92 & 40.7567 \\
\hline & WAFSA-BPANN & 0.3055 & 0.1601 & 6.87 & 146.8292 \\
\hline
\end{tabular}

TABLE 7: Errors of different traditional models in June.

\begin{tabular}{|c|c|c|c|c|c|}
\hline & Models & $\operatorname{MAE}(\mathrm{m} / \mathrm{s})$ & $\operatorname{MSE}\left(\mathrm{m}^{2} / \mathrm{s}^{2}\right)$ & MAPE (\%) & Running time (s) \\
\hline \multirow{6}{*}{ Observation site 1} & BPANN & 0.4637 & 0.4260 & 10.48 & 0.8509 \\
\hline & ARIMA & 0.4617 & 0.4215 & 10.06 & 37.0557 \\
\hline & AFSA-BPANN & 0.4701 & 0.4267 & 10.91 & 104.7545 \\
\hline & WT-BPANN & 0.4625 & 0.4278 & 10.05 & 1.4735 \\
\hline & WT-ARIMA & 0.4663 & 0.4315 & 10.08 & 37.9540 \\
\hline & WAFSA-BPANN & 0.3381 & 0.2066 & 8.14 & 145.9524 \\
\hline \multirow{6}{*}{ Observation site 2} & BPANN & 0.4446 & 0.3783 & 9.99 & 1.1537 \\
\hline & ARIMA & 0.4442 & 0.3737 & 9.78 & 41.4958 \\
\hline & AFSA-BPANN & 0.4443 & 0.3770 & 10.07 & 108.9195 \\
\hline & WT-BPANN & 0.4450 & 0.3849 & 9.76 & 73.93 \\
\hline & WT-ARIMA & 0.4462 & 0.3859 & 9.78 & 42.0491 \\
\hline & WAFSA-BPANN & 0.3441 & 0.2084 & 8.27 & 143.1720 \\
\hline \multirow{6}{*}{ Observation site 3} & BPANN & 0.4716 & 0.3987 & 11.78 & 0.5463 \\
\hline & ARIMA & 0.4671 & 0.3930 & 11.09 & 36.7596 \\
\hline & AFSA-BPANN & 0.4720 & 0.3989 & 11.7 & 104.6663 \\
\hline & WT-BPANN & 0.4700 & 0.4144 & 11.17 & 0.7220 \\
\hline & WT-ARIMA & 0.4727 & 0.4198 & 11.08 & 41.9609 \\
\hline & WAFSA-BPANN & 0.3353 & 0.2008 & 8.64 & 142.3210 \\
\hline
\end{tabular}

MA: Moving Average Model

MAE: Mean absolute error

MAPE: Mean absolute percentage error

MSE: Mean square error

NWPs: Numerical Weather Prediction Systems

NEA: National energy administration

PHEVs: Plug-in hybrid electric vehicles
RBF: Radial Basis Function

RES: Renewable energy source WT: Wavelet transform.

\section{Conflict of Interests}

The authors declare that there is no conflict of interests regarding the publication of this paper. 
TABLE 8: Errors of different traditional models in August.

\begin{tabular}{|c|c|c|c|c|c|}
\hline & Models & $\operatorname{MAE}(\mathrm{m} / \mathrm{s})$ & $\operatorname{MSE}\left(\mathrm{m}^{2} / \mathrm{s}^{2}\right)$ & MAPE (\%) & Running time (s) \\
\hline \multirow{6}{*}{ Observation site 1} & BPANN & 0.4595 & 0.3856 & 7.95 & 1.0696 \\
\hline & ARIMA & 0.4657 & 0.3989 & 8.05 & 39.0358 \\
\hline & AFSA-BPANN & 0.4612 & 0.3840 & 7.98 & 102.2733 \\
\hline & WT-BPANN & 0.4668 & 0.3956 & 8.05 & 1.0735 \\
\hline & WT-ARIMA & 0.4787 & 0.4201 & 8.25 & 43.0450 \\
\hline & WAFSA-BPANN & 0.3377 & 0.2055 & 5.94 & 147.2995 \\
\hline \multirow{6}{*}{ Observation site 2} & BPANN & 0.4189 & 0.3351 & 8.12 & 0.4442 \\
\hline & ARIMA & 0.4189 & 0.3396 & 8.13 & 37.0051 \\
\hline & AFSA-BPANN & 0.4139 & 0.3259 & 8 & 105.3182 \\
\hline & WT-BPANN & 0.4239 & 0.3438 & 8.17 & 0.9281 \\
\hline & WT-ARIMA & 0.4303 & 0.3563 & 8.3 & 37.1158 \\
\hline & WAFSA-BPANN & 0.3043 & 0.1831 & 6.07 & 145.4899 \\
\hline \multirow{6}{*}{ Observation site 3} & BPANN & 0.4601 & 0.3883 & 8.26 & 2.2823 \\
\hline & ARIMA & 0.4689 & 0.3981 & 8.48 & 37.7879 \\
\hline & AFSA-BPANN & 0.4600 & 0.3850 & 8.32 & 102.6349 \\
\hline & WT-BPANN & 0.4665 & 0.3928 & 8.39 & 2.0385 \\
\hline & WT-ARIMA & 0.4727 & 0.4044 & 8.48 & 40.7267 \\
\hline & WAFSA-BPANN & 0.3407 & 02140 & 6.35 & 142.8692 \\
\hline
\end{tabular}

TABLE 9: Errors of different traditional models in October.

\begin{tabular}{|c|c|c|c|c|c|}
\hline & Models & $\operatorname{MAE}(\mathrm{m} / \mathrm{s})$ & $\operatorname{MSE}\left(\mathrm{m}^{2} / \mathrm{s}^{2}\right)$ & MAPE (\%) & Running time (s) \\
\hline \multirow{6}{*}{ Observation site 1} & BPANN & 0.3824 & 0.2768 & 7.2 & 0.5651 \\
\hline & ARIMA & 0.3827 & 0.2766 & 7.18 & 32.6927 \\
\hline & AFSA-BPANN & 0.3827 & 0.2752 & 7.22 & 110.4872 \\
\hline & WT-BPANN & 0.3898 & 0.2954 & 7.25 & 0.6419 \\
\hline & WT-ARIMA & 0.3930 & 0.3019 & 7.27 & 35.1270 \\
\hline & WAFSA-BPANN & 0.2963 & 0.1503 & 5.79 & 151.7133 \\
\hline \multirow{6}{*}{ Observation site 2} & BPANN & 0.4141 & 0.3206 & 8.05 & 0.8032 \\
\hline & ARIMA & 0.4122 & 0.3257 & 7.89 & 33.0404 \\
\hline & AFSA-BPANN & 0.4157 & 0.3238 & 8.11 & 109.5214 \\
\hline & WT-BPANN & 0.4274 & 0.3511 & 8.11 & 0.8312 \\
\hline & WT-ARIMA & 0.4301 & 0.3526 & 8.16 & 35.2028 \\
\hline & WAFSA-BPANN & 0.3164 & 0.1983 & 6.34 & 147.8061 \\
\hline \multirow{6}{*}{ Observation site 3} & BPANN & 0.4156 & 0.3245 & 8.75 & 0.5211 \\
\hline & ARIMA & 0.4148 & 0.3242 & 8.85 & 35.9502 \\
\hline & AFSA-BPANN & 0.4085 & 0.3132 & 8.78 & 105.3837 \\
\hline & WT-BPANN & 0.4167 & 0.3288 & 8.9 & 0.5227 \\
\hline & WT-ARIMA & 0.4297 & 0.3504 & 9.15 & 36.1737 \\
\hline & WAFSA-BPANN & 0.3356 & 0.2173 & 7.45 & 142.9675 \\
\hline
\end{tabular}

TABLE 10: Comprehensive comparison of the performances of models involved.

\begin{tabular}{lccccccc}
\hline Model & $\begin{array}{c}\text { MAE } \\
(\mathrm{m} / \mathrm{s})\end{array}$ & $\begin{array}{c}\text { Improvement } \\
(\%)\end{array}$ & $\begin{array}{c}\text { MSE } \\
\left(\mathrm{m}^{2} / \mathrm{s}^{2}\right)\end{array}$ & $\begin{array}{c}\text { Improvement } \\
(\%)\end{array}$ & $\begin{array}{c}\text { MAPE } \\
(\%)\end{array}$ & $\begin{array}{c}\text { Improvement } \\
(\%)\end{array}$ & $\begin{array}{c}\text { Running time } \\
(\mathrm{s})\end{array}$ \\
\hline BPANN & 0.4284 & $\mathbf{2 4 . 7 0}$ & 0.3461 & $\mathbf{4 5 . 2 5}$ & 8.7525 & $\mathbf{2 1 . 2 8}$ & 1.14 \\
ARIMA & 0.4330 & $\mathbf{2 5 . 5 0}$ & 0.3522 & $\mathbf{4 6 . 2 0}$ & 8.74 & $\mathbf{2 1 . 1 7}$ & 38.0052 \\
AFSA-BPANN & 0.4289 & $\mathbf{2 4 . 7 8}$ & 0.3446 & $\mathbf{4 5 . 0 1}$ & 8.8183 & $\mathbf{2 1 . 8 7}$ & 105.9424 \\
WT-BPANN & 0.4281 & $\mathbf{2 4 . 6 4}$ & 0.3482 & $\mathbf{4 5 . 5 8}$ & 8.6233 & $\mathbf{2 0 . 1}$ & 1.4398 \\
WT-ARIMA & 0.4332 & $\mathbf{2 5 . 5 3}$ & 0.3571 & $\mathbf{4 6 . 9 3}$ & 8.6691 & $\mathbf{2 0 . 5 2}$ & 39.4098 \\
WAFSA-BPANN & $\mathbf{0 . 3 2 2 6}$ & - & $\mathbf{0 . 1 8 9 5}$ & - & $\mathbf{6 . 8 9}$ & - & 146.8031 \\
\hline
\end{tabular}




\section{Acknowledgments}

This work was funded by the Support Plan for Leaders of the First-class Discipline with Characteristics in Colleges and Universities of Liaoning Province of China under Grant (XKRC-201403) and the project of DUFE research base (DUFE2014J29), the modeling and analysis of the inflow and outflow of full-caliber regional water sources of China.

\section{References}

[1] J.-M. Hu, J.-Z. Wang, and G.-W. Zeng, "A hybrid forecasting approach applied to wind speed time series," Renewable Energy, vol. 60, pp. 185-194, 2013.

[2] A. J. Lamadrid, "Optimal use of energy storage systems with renewable energy sources," International Journal of Electrical Power \& Energy Systems, vol. 71, pp. 101-111, 2015.

[3] X. Luo, J. Wang, M. Dooner, and J. Clarke, "Overview of current development in electrical energy storage technologies and the application potential in power system operation," Applied Energy, vol. 137, pp. 511-536, 2015.

[4] L. Zhang, Z. Wang, F. Sun, and D. G. Dorrell, "Online parameter identification of ultracapacitor models using the extended Kalman filter," Energies, vol. 7, no. 5, pp. 3204-3217, 2014.

[5] N. Juul and P. Meibom, "Road transport and power system scenarios for Northern Europe in 2030," Applied Energy, vol. 92, pp. 573-582, 2012.

[6] T. Takeshita, "Assessing the co-benefits of $\mathrm{CO}_{2}$ mitigation on air pollutants emissions from road vehicles," Applied Energy, vol. 97, pp. 225-237, 2012.

[7] D. Kum, H. Peng, and N. K. Bucknor, "Optimal control of plugin HEVs for fuel economy under various travel distances," in Proceedings of the 6th IFAC Symposium Advances in Automotive Control (AAC '10), pp. 258-263, Munich, Germany, July 2010.

[8] W. Sung and C. B. Shin, "Electrochemical model of a lithiumion battery implemented into an automotive battery management system," Computers \& Chemical Engineering, vol. 76, pp. 87-97, 2015.

[9] B. Johansson and A. Mårtensson, "Energy and environmental costs for electric vehicles using $\mathrm{CO}_{2}$-neutral electricity in Sweden," Energy, vol. 25, no. 8, pp. 777-792, 2000.

[10] Y. Kudoh, H. Ishitani, R. Matsuhashi et al., "Environmental evaluation of introducing electric vehicles using a dynamic traffic-flow model," Applied Energy, vol. 69, no. 2, pp. 145-159, 2001.

[11] D. MeCimorec and Z. Tomsic, "Portfolio theory application in wind potential assessment," Renewable Energy, vol. 76, pp. 494502, 2015.

[12] X.-C. Wang, P. Guo, and X.-B. Huang, "A review of wind power forecasting models," Energy Procedia, vol. 12, pp. 770-778, 2011.

[13] J. Z. Wang, S. S. Qin, Q. P. Zhou, and H. Y. Jiang, "Mediumterm wind speeds forecasting utilizing hybrid models for three different sites in Xinjiang, China," Renewable Energy, vol. 76, pp. 91-101, 2015.

[14] C. Croonenbroeck and D. Ambach, "A selection of time series models for short- to medium-term wind power forecasting," Journal of Wind Engineering and Industrial Aerodynamics, vol. 136, pp. 201-210, 2015.

[15] Y. Zhang, J.-X. Wang, and X.-F. Wang, "Review on probabilistic forecasting of wind power generation," Renewable and Sustainable Energy Reviews, vol. 32, pp. 255-270, 2014.
[16] J. Szoplik, "Forecasting of natural gas consumption with artificial neural networks," Energy, vol. 85, pp. 208-220, 2015.

[17] Y.-Y. Hong, H.-L. Chang, and C.-S. Chiu, "Hour-ahead wind power and speed forecasting using simultaneous perturbation stochastic approximation (SPSA) algorithm and neural network with fuzzy inputs," Energy, vol. 35, no. 9, pp. 3870-3876, 2010.

[18] B. Ernst, B. Oakleaf, M. L. Ahlstrom et al., "Predicting the wind," IEEE Power and Energy Magazine, vol. 5, no. 6, pp. 78-89, 2007.

[19] S. Baran, "Probabilistic wind speed forecasting using Bayesian model averaging with truncated normal components," Computational Statistics \& Data Analysis, vol. 75, pp. 227-238, 2014.

[20] G. Sideratos and N. D. Hatziargyriou, "An advanced statistical method for wind power forecasting," IEEE Transactions on Power Systems, vol. 22, no. 1, pp. 258-265, 2007.

[21] S. A. Kalogirou, "Applications of artificial neural-networks for energy systems," Applied Energy, vol. 67, no. 1-2, pp. 17-35, 2000.

[22] J. C. Spall, "Multivariate stochastic approximation using a simultaneous perturbation gradient approximation," IEEE Transactions on Automatic Control, vol. 37, no. 3, pp. 332-341, 1992.

[23] W.-Y. Liu and J.-G. Han, "The optimal Mexican hat wavelet filter de-noising method based on cross-validation method," Neurocomputing, vol. 108, no. 2, pp. 31-35, 2013.

[24] A. Bakhtazad, A. Palazoglu, and J. A. Romagnoli, "Process data de-noising using wavelet transform," Intelligent Data Analysis, vol. 3, no. 4, pp. 267-285, 1999.

[25] H. Liu, H.-Q. Tian, Y.-F. Li, and L. Zhang, "Comparison of four Adaboost algorithm based artificial neural networks in wind speed predictions," Energy Conversion and Management, vol. 92, pp. 67-81, 2015.

[26] H. D. Tran, N. Muttil, and B. J. C. Perera, "Selection of significant input variables for time series forecasting," Environmental Modelling \& Software, vol. 64, pp. 156-163, 2015.

[27] M. Ghofrani, M. Ghayekhloo, A. Arabali, and A. Ghayekhloo, "A hybrid short-term load forecasting with a new input selection framework," Energy, vol. 81, pp. 777-786, 2015.

[28] R. Hecht-Nielsen, "Kolmogorov's mapping neural network existence theorem," in Proceedings of the International Conference on Neural Networks, 1987.

[29] A. Suresh, K. V. Harish, and N. Radhika, "Particle swarm optimization over back propagation neural network for length of stay prediction," Procedia Computer Science, vol. 46, pp. 268 275, 2015.

[30] X.-L. Li, Z.-J. Shao, and J.-X. Qian, "An optimizing method based on autonomous animats: fish-swarm Algorithm," System Engineering Theory and Practice, vol. 22, no. 11, pp. 32-38, 2002.

[31] N. Fang, J.-Z. Zhou, R. Zhang, Y. Liu, and Y.-C. Zhang, "A hybrid of real coded genetic algorithm and artificial fish swarm algorithm for short-term optimal hydrothermal scheduling," International Journal of Electrical Power \& Energy Systems, vol. 62, pp. 617-629, 2014.

[32] Z. Wu and N. E. Huang, "Ensemble empirical mode decomposition: a noise-assisted data analysis method," Advances in Adaptive Data Analysis, vol. 1, no. 1, pp. 1-41, 2009.

[33] S. S. Qin, F. Liu, J.-Z. Wang, and Y.-L. Song, "Interval forecasts of a novelty hybrid model for wind speeds," Energy Reports, vol. 1, pp. 8-16, 2015. 


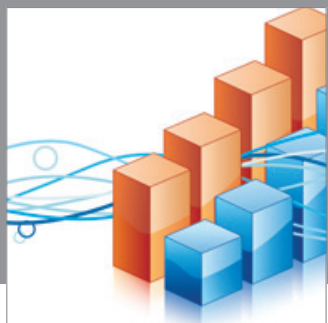

Advances in

Operations Research

mansans

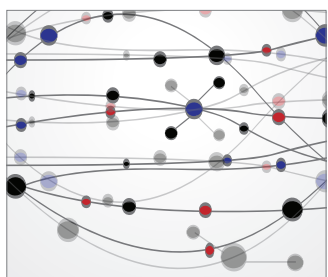

The Scientific World Journal
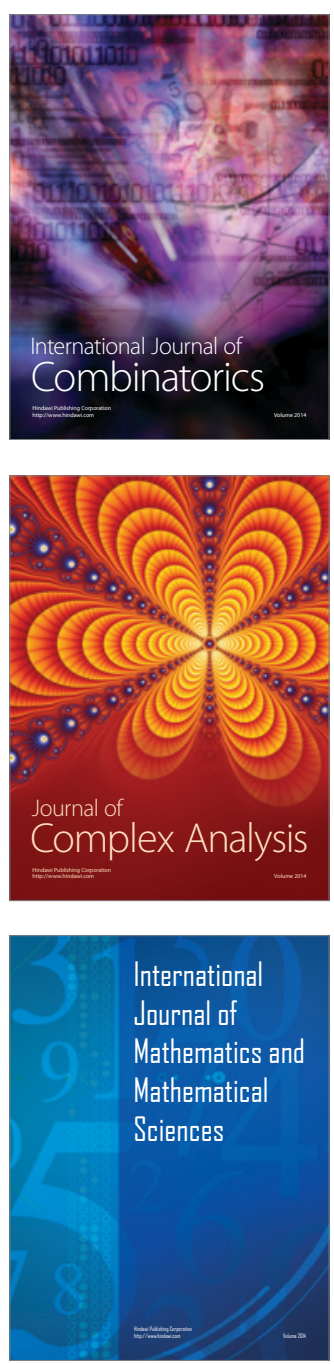
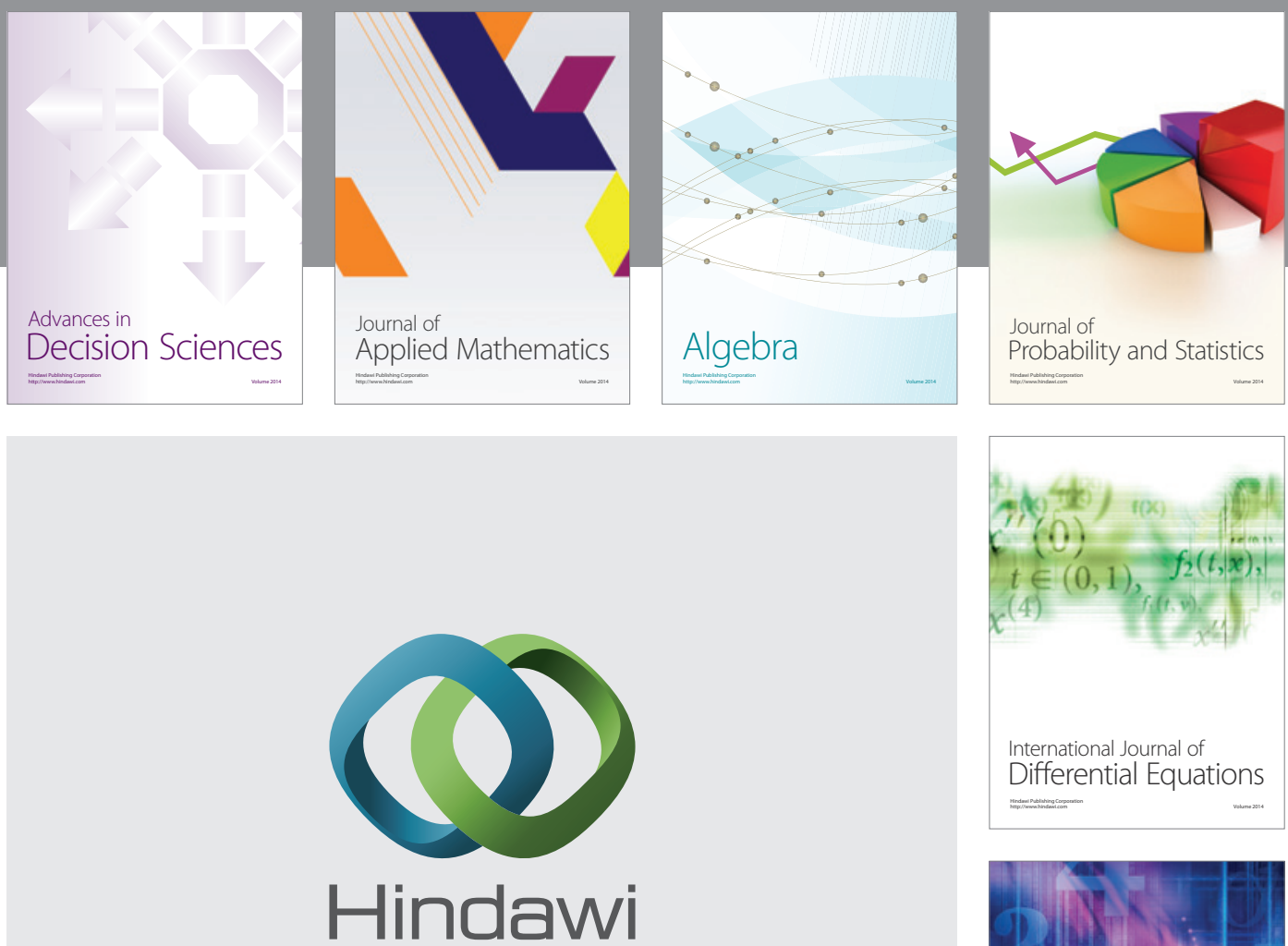

Submit your manuscripts at http://www.hindawi.com
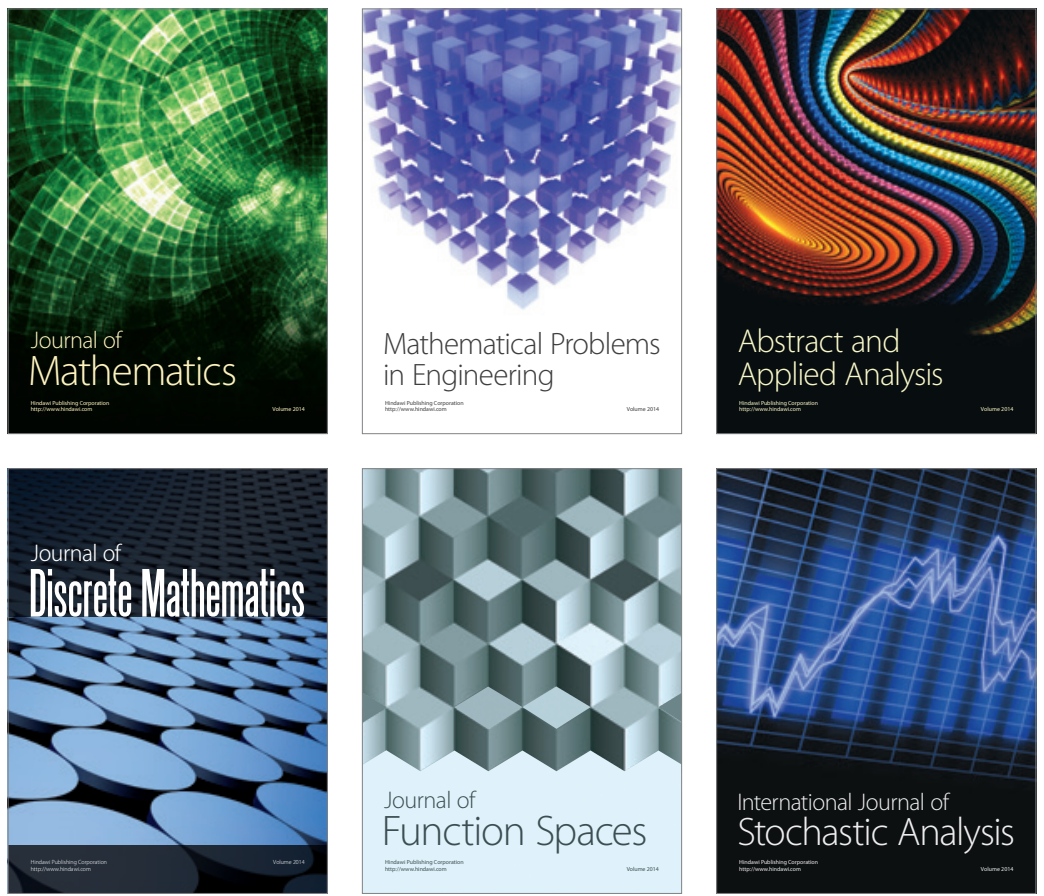

Journal of

Function Spaces

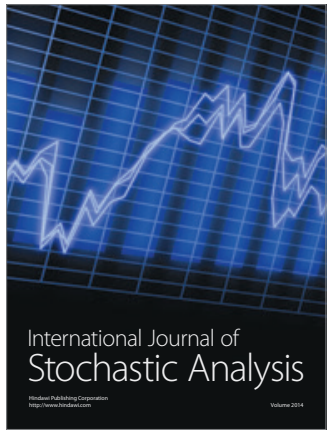

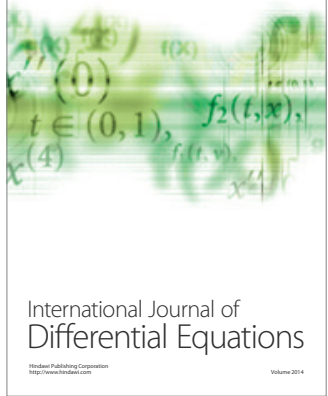
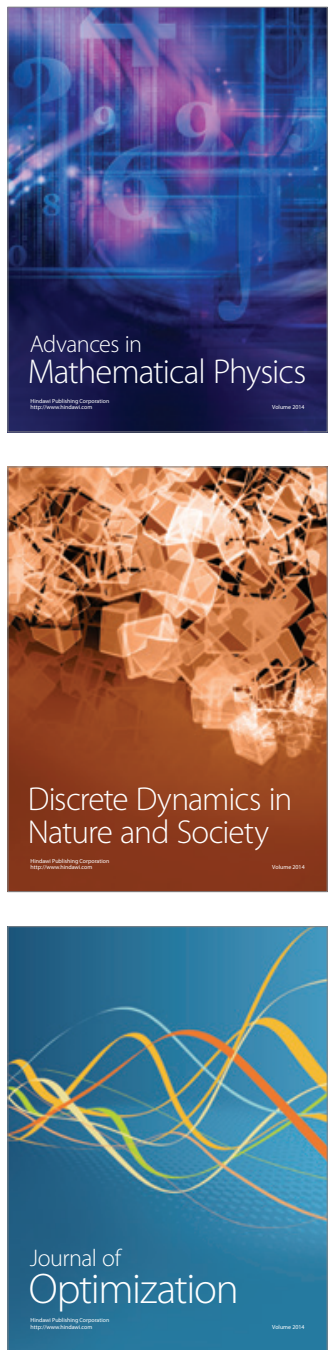